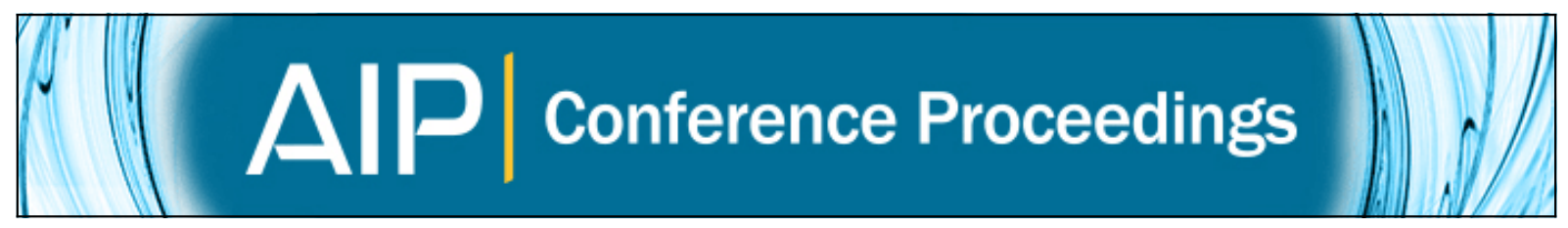

\title{
Foreground Science Knowledge and Prospects
}

Aurélien A. Fraisse, Jo-Anne C. Brown, Gregory Dobler, Jessie L. Dotson, Bruce T. Draine, Priscilla C. Frisch, Marijke Haverkorn, Christopher M. Hirata, Ronnie Jansson, Alex Lazarian, Antonio Mario Magalhães, André Waelkens, and Maik Wolleben

Citation: AIP Conference Proceedings 1141, 265 (2009); doi: 10.1063/1.3160889

View online: http://dx.doi.org/10.1063/1.3160889

View Table of Contents: http://scitation.aip.org/content/aip/proceeding/aipcp/1141?ver=pdfcov

Published by the AIP Publishing

\section{Articles you may be interested in}

Can the near-IR fluctuations arise from known galaxy populations?

AIP Conf. Proc. 1480, 370 (2012); 10.1063/1.4754390

Prospects for polarized foreground removal

AIP Conf. Proc. 1141, 222 (2009); 10.1063/1.3160888

Reionization Science with the Cosmic Microwave Background

AIP Conf. Proc. 1141, 179 (2009); 10.1063/1.3160887

Simulating Reionization: Character and Observability

AIP Conf. Proc. 990, 442 (2008); 10.1063/1.2905659

Evolving stellar background radiation and gamma-ray optical depth

AIP Conf. Proc. 558, 862 (2001); 10.1063/1.1370894 


\title{
Foreground Science Knowledge and Prospects
}

Aurélien A. Fraisse ${ }^{\mathrm{a}, *}$, Jo-Anne C. Brown ${ }^{\mathrm{b}}$, Gregory Dobler ${ }^{\mathrm{c}}$, Jessie L. Dotson ${ }^{\mathrm{d}}$, Bruce T. Draine $^{\mathrm{a}}$, Priscilla C. Frisch ${ }^{\mathrm{e}}$, Marijke Haverkorn ${ }^{\mathrm{f}, \mathrm{g}, \mathrm{h}}$, Christopher M. Hirata ${ }^{\mathrm{i}}$, Ronnie Jansson ${ }^{\mathrm{j}}$, Alex Lazarian ${ }^{\mathrm{k}}$, Antonio Mario Magalhães ${ }^{\mathrm{l}}$, André Waelkens ${ }^{\mathrm{m}}$, and Maik Wolleben ${ }^{\mathrm{n}}$

${ }^{a}$ Princeton University Observatory, Peyton Hall, Princeton, NJ 08544, USA

${ }^{\mathrm{b}}$ Centre for Radio Astronomy, University of Calgary, Calgary, AB T2N 1N4, Canada

${ }^{\mathrm{C}}$ Harvard-Smithsonian Center for Astrophysics, 60 Garden Street, Cambridge, MA 02138, USA

${ }^{\mathrm{d}}$ NASA Ames Research Center, Moffet Field, CA 94035, USA

${ }^{\mathrm{e}}$ University of Chicago, 5460 South Ellis Avenue, Chicago, IL 60637, USA

${ }^{\mathrm{f}}$ Jansky Fellow, National Radio Astronomy Observatory, Charlottesville, VA 22903, USA

${ }^{\circ}$ Astronomy Department, UC Berkeley, 601 Campbell Hall, Berkeley, CA 94720, USA

${ }^{\mathrm{h}}$ ASTRON, P.O. Box 2, 7990 AA Dwingeloo, the Netherlands

${ }^{\mathrm{i}}$ Caltech, Mail Code 130-33, Pasadena, CA 91125, USA

${ }^{\mathrm{j} C e n t e r}$ for Cosmology and Particle Physics, Department of Physics, NYU, NY, NY 10003, USA

${ }^{\mathrm{k}}$ Department of Astronomy, University of Wisconsin-Madison, Madison, WI 53706, USA

${ }^{1} I A G$, Universidade de São Paulo, Rua do Matão 1226, São Paulo, SP 05508-900, Brazil

${ }^{\mathrm{m}}$ Max-Planck-Institut für Astrophysik, Garching 85\%41, Germany

${ }^{\mathrm{n}}$ Covington Fellow, NRC Herzberg Institute of Astrophysics, DRAO, Pentic-

ton, BV V2A 6J9, Canada

* fraisse@astro.princeton.edu

\begin{abstract}
Detecting "B-mode" (i.e., divergence free) polarization in the Cosmic Microwave Background (CMB) would open a new window on the very early Universe. However, the polarized microwave sky is dominated by polarized Galactic dust and synchrotron emissions, which may hinder our ability to test inflationary predictions. In this paper, we report on our knowledge of these "Galactic foregrounds," as well as on how a CMB satellite mission aiming at detecting a primordial B-mode signal ("CMBPol") will contribute to improving it. We review the observational and analysis techniques used to constrain the structure of the Galactic magnetic field, whose presence is responsible for the polarization of Galactic emissions. Although our current understanding of the magnetized interstellar medium is somewhat limited, dramatic improvements in our knowledge of its properties are expected by the time CMBPol flies. Thanks to high resolution and high sensitivity instruments observing the whole sky at frequencies between $30 \mathrm{GHz}$ and $850 \mathrm{GHz}, \mathrm{CMBPol}$ will not only improve this picture by observing the synchrotron emission from our galaxy, but also help constrain
\end{abstract}

CP1141, CMB Polarizaiton Workshop: Theory and Foregrounds, CMBPol Mission Concept Study edited by S. Dodelson, D. Baumann, A. Cooray, J. Dunkley, A. A. Fraisse, M. G. Jackson, A. Kogut, L. M. Krauss, K. M. Smith, and M. Zaldarriaga 2009 American Institute of Physics 978-0-7354-0678-0/09/\$25.00 
dust models. Polarized emission from interstellar dust indeed dominates over any other signal in CMBPol's highest frequency channels. Observations at these wavelengths, combined with groundbased studies of starlight polarization, will therefore enable us to improve our understanding of dust properties and of the mechanism(s) responsible for the alignment of dust grains with the Galactic magnetic field. CMBPol will also shed new light on observations that are presently not well understood. Morphological studies of anomalous dust and synchrotron emissions will indeed constrain their natures and properties, while searching for fluctuations in the emission from heliospheric dust will test our understanding of the circumheliospheric interstellar medium. Finally, acquiring more information on the properties of extra-Galactic sources will be necessary in order to maximize the cosmological constraints extracted from CMBPol's observations of CMB lensing.

\section{Introduction}

Over the course of the last two decades, studies of the Cosmic Microwave Background (CMB) have revolutionized our understanding of the composition, structure and evolution of the Universe. After sixteen years of observations of the CMB temperature anisotropies first detected by the COBE satellite in the early 1990's (Smoot et al., 1992), we now have a standard cosmological model fully described by a handful of parameters, and in spectacular agreement with a broad range of independent astrophysical observations (Komatsu et al., 2008).

The cornerstone of this achievement is doubtless inflation. This paradigm, initially designed to explain the observed flatness and isotropy of the Universe (Guth, 1981), provides a natural explanation for several of its other properties, including the origin of the primordial density fluctuations that led to structure formation. A generic prediction of the most natural inflationary models is the existence of primordial gravitational waves that should 
have left their imprint on the CMB anisotropies in the form of a divergence-free polarization pattern known as "B-mode polarization." However, this signal is expected to have a very low amplitude, typically more than three to four orders of magnitude smaller than the measured CMB temperature fluctuations (for a review of inflationary predictions, see the companion paper by Baumann et al., 2008a). Detecting primordial B-modes is therefore a challenging enterprise made only more difficult by polarized emissions from our galaxy and others. Among these so-called "polarized foregrounds" are polarized synchrotron and dust emissions from the Galaxy, which dominate the expected primordial B-mode signal over the whole sky at all frequencies of interest (see, e.g., the discussion in Page et al., 2007). Thus, being able to properly handle them is central to being able to detect low amplitude B-modes in the CMB.

In this document, we do not address the question of how to look behind this Galactic (and extra-Galactic) screen, but focus on our knowledge of the foregrounds themselves. Our ability to use the data provided by a satellite mission sensitive enough to detect Bmodes of inflationary origin (hereafter, $\mathrm{CMBPol}$ ) is indeed limited by our understanding of foregrounds (see the companion paper by Dunkley et al., 2008a). We therefore review what we know now, how we know it, and how we hope to improve it by the time CMBPol flies. Moreover, since Galactic foregrounds are the dominant signal CMBPol will observe, we can expect our understanding of these emissions and their underlying physical processes to significantly improve through an analysis of the CMBPol data. Although our current knowledge of foregrounds makes it difficult to quantify many of these possible improvements, we review what we think are the main ways in which CMBPol will help us explore the magnetized Galactic interstellar medium.

Four companion papers complement the contents of this document: Baumann et al. (2008a) review the inflationary science case for CMBPol; Smith et al. (2008) report on CMB lensing; Zaldarriaga et al. (2008) discuss reionization; and Dunkley et al. (2008a) provide a detailed analysis of how and how well a primordial B-mode signal can be separated from the polarized emissions from the Galaxy. An executive summary of these documents is also available (Baumann et al., 2008b). For the purposes of this paper and all companion documents, we assume that CMBPol will have specifications close to those provided in Tab. 1, which fall between the specifications of the two EPIC designs proposed in the Bock et al. (2008) report.

Table 1: Possible CMBPol ${ }^{\mathrm{a}}$ Specifications

\begin{tabular}{ll|c|c|c|c|c|c|c|c|c}
\hline \hline Frequency & (GHz) & 30 & 45 & 70 & 100 & 150 & 220 & 340 & 500 & 850 \\
\hline Resolution & (FWHM in arcmin) & 26 & 17 & 11 & 8 & 5 & 3.5 & 2.3 & 1.5 & 0.9 \\
\hline Sensitivity $^{\mathrm{b}}$ & (nK CMB) & 160 & 69 & 35 & 27 & 26 & 40 & 180 & $150^{\mathrm{c}}$ & $400^{\mathrm{d}}$ \\
\hline \hline
\end{tabular}

${ }^{a}$ Expected mission lifetime of 4 years

${ }^{\mathrm{b}} \mathrm{CMB}$ temperature fluctuations detectable at $1 \sigma$ in a $2^{\circ} \times 2^{\circ}$ pixel

${ }^{\mathrm{c}}$ Could be improved by a factor of 5 by cooling the telescope to $4 \mathrm{~K}$

${ }^{\mathrm{d}}$ Could be improved by a factor of 40 by cooling the telescope to $4 \mathrm{~K}$ 
This document is organized as follows. In Sec. 2, we review the observational techniques used to probe the Galactic magnetic field (Sec. 2.1), as well as what they teach us about its main properties (Sec. 2.2), while Sec. 3 presents an analysis technique aimed at improving our constraints on the magnetized interstellar medium. Sec. 4 discusses the progress one can expect from upcoming radio (Sec. 4.1) and CMB (Sec. 4.2) observations, including those by CMBPol. Our knowledge of the polarized emission from interstellar dust is then summarized in Sec. 5, and followed by a description of the physical mechanism responsible for it in Sec. 6. Heliospheric dust emission, and "anomalous emissions" detected in the WMAP data, are the topics of, respectively, Sec. 7 and Sec. 8. Finally, Sec. 9 gives an overview of the foreground situation in the context of CMB lensing studies, and our conclusions are presented in Sec. 10.

\section{Probing the Galactic Magnetic Field}

The idea that the Galaxy has a magnetic field, and that it may play an important role in Galactic physics, dates back to Fermi (1949). He proposed that cosmic rays might be generated outside of the solar system as a result of energetic particles colliding with moving irregularities in this field. In his view, the magnetic field would not only be a cosmic ray generator, but also a containment factor preventing the rays from escaping the Galaxy.

It is now believed that magnetic fields and cosmic rays indeed contribute to the vertical support of the gas in the Galaxy, with the magnetic field energy density dominating the turbulent and thermal gas energy densities at high Galactic latitudes (Boulares \& Cox, 1990). However, astronomical magnetic fields also play important roles in many other processes affecting the structure of the Galaxy. In star formation, they can inhibit the gravitational collapse of interstellar clouds (primary star formation regions) and help removing prestellar angular momentum (Zweibel \& Heiles, 1997), thereby affecting the distribution of stars in the Galaxy. It is also believed that magnetic fields influence galaxy formation and evolution by causing large density fluctuations which result in structures within galaxies (Kim et al., 1996).

Yet despite the Galactic magnetic field's importance, many mysteries remain about its generation and evolution. A dynamo mechanism is believed to be necessary to increase galactic magnetic field strengths from that of a tiny primordial seed field to the observed values (see, e.g., Beck et al., 1996), but neither the exact dynamo mechanism nor the generation and strength of the seed field are significantly constrained by available observations. External galaxies appear to have a wide range of magnetic field characteristics (Beck, 2001), indicating that different dynamos must be acting in different galaxies. Furthermore, in some cases, rapid field amplification seems to be required (Gaensler et al., 2005). Before we can hope to fully explore these aspects of the field, we must first understand the field's general topology.

While observations of polarized emission from interstellar dust grains, polarization of starlight, and Zeeman splitting can provide in depth probing of the field in localized regions, most of the knowledge we have about the magnetic field across the bulk of the Galaxy comes from radio observations of linearly-polarized radiation. At frequencies above a few $\mathrm{GHz}$, 
maps of radio polarization angles directly yield the direction of the magnetic field component in the plane of the sky, while the total emission indicates its strength. At lower frequencies, Faraday rotation of polarized emission traveling through the interstellar medium (ISM) can be used to probe the magnetic field component parallel to the line-of-sight. We discuss these techniques in Sec. 2.1, before detailing the insights gained from these observations in Sec. 2.2 .

\subsection{Observational Techniques}

\subsubsection{Radio Synchrotron Radiation}

Relativistic electrons moving in the Galactic magnetic field emit synchrotron radiation. Optically thin synchrotron emission, $\epsilon_{\mathrm{s}}$, at frequency $\nu$ depends on the component of the uni-

form magnetic field perpendicular to the line-of-sight, $B_{\perp}$, according to $\epsilon_{\mathrm{S}} \propto B_{\perp}{ }^{(p+1) / 2} \nu^{-(p-1) / 2}$ (Rybicki \& Lightman, 1979). The exponent $p$ describes the power law energy spectrum of the ensemble of relativistic electrons through the relation $N(E) \propto E^{-p}$. This emission therefore provides information about the slope of the electron energy spectrum, as well as an estimate of the strength of the magnetic field if assumptions are made about the volume of the source and the energy density of cosmic rays. The minimum combined energy density of cosmic ray particles and magnetic field necessary to reproduce the emission from an observed source is similar to the equipartition energy density between magnetic field and cosmic rays. Magnetic field estimates from the brightness of synchrotron emission usually assume this minimum energy condition, or equipartition, though often without clear observational evidence (through $\gamma$ - or X-ray data) that it applies (see, e.g., the discussion in Beck \& Krause, 2005).

Synchrotron emission from a region with a uniform magnetic field and an electron distribution with $p=3$ (respectively, $p=2$ ) has a theoretical polarization of $75 \%$ (respectively, $60 \%$ ), with the plane of polarization perpendicular to the direction of $B_{\perp}$ in the plane of the sky (Rybicki \& Lightman, 1979). Polarization of synchrotron emission therefore also gives information on the magnetic field component perpendicular to the line-of-sight. In practice, the observed emission is integrated over large regions in space with a complicated magnetic field structure and an ionized plasma present in addition to the relativistic electrons. Consequently, the integrated emission is usually much less polarized than the theoretical limit.

Although maps of the synchrotron emission can inform us on the strength and direction of the component of the Galactic magnetic field perpendicular to the line-of-sight, they do not encode any information related to the component parallel to the line-of-sight. The latter can be provided by Faraday rotation studies (Sec. 2.1.2) or rotation measure synthesis (Sec. 2.1.3).

\subsubsection{Faraday Rotation of Compact Sources}

When a linearly polarized electromagnetic wave propagates through a region of free electrons permeated by a magnetic field (e.g., a magnetized plasma such as the ISM), its plane of 
polarization rotates. In the quasi-longitudinal (QL) approximation (Ratcliffe, 1959), which assumes the field direction is roughly along the line-of-sight, the Faraday rotation angle (in radians) at wavelength $\lambda$ (in meters) is $\Psi \simeq \lambda^{2} \mathrm{RM}$, where $\mathrm{RM}$, the "rotation measure," is

$$
\mathrm{RM} \equiv 0.812 \int n_{\mathrm{e}} \mathbf{B} \cdot \mathrm{d} \mathbf{l}\left(\mathrm{rad} \mathrm{m}^{-2}\right)
$$

with $n_{\mathrm{e}}$ the electron density in units of $\mathrm{cm}^{-3}, \mathbf{B}$ the magnetic field in units of $\mu \mathrm{G}$, and dl the incremental pathlength from the source to the receiver in units of pc. At radio frequencies, with typical Galactic magnetic field strengths and electron densities, the QL approximation can be shown to hold for fields which are essentially perpendicular to the line-of-sight. Consequently, Eq. (1) is actually valid for most ISM applications.

If we assume that at all wavelengths the polarized emission from a given source is emitted at the same initial polarization angle $\varphi_{\mathrm{o}}$, and that the radiation is only affected by Faraday rotation, the detected polarization angle $\varphi$ is given by the linear relationship $\varphi=\varphi_{0}+\lambda^{2}$ RM. Measurements of $\varphi$ at multiple wavelengths can therefore determine the $\mathrm{RM}$ for a given source as the slope of the graph of $\varphi$ vs. $\lambda^{2}$. In practice, at least three frequency channels are needed to properly do this exercise. If many more are available, RMs may also be calculated by using a RM transfer function (RMTF) (see Brentjens \& de Bruyn, 2005, and Sec. 2.1.3).

The simplicity with which RMs can be determined, coupled with the significance of their signs, makes them powerful tools with which one can probe the ISM magnetic field. Pulsars and extragalactic sources (EGS) emit linearly polarized radiation and are typically used to study the Galactic magnetic field. Since it is possible to estimate their distances to us, we can work backwards and find out what the field looks like along lines-of-sight to them, provided we make some assumptions about the electron density distribution within the Galaxy.

When pulsars are used, studying the dispersion of the emitted pulses provides information to replace some of these assumptions. Different frequencies propagate at different group velocities in the interstellar medium. As a result, a pulse emitted by a given pulsar is not received at the same time at different frequencies. The arrival time $\tau$ at frequency $\nu$ is such that

$$
\frac{\mathrm{d} \tau}{\mathrm{d} \nu} \propto-\frac{1}{\nu^{3}} \int n_{\mathrm{e}} \mathrm{d} \mathbf{l}
$$

which is a measurable quantity. Since the positive proportionality constant is a combination of known physical constants, it is possible to compute the value of the integral, called dispersion measure (DM), and therefore to estimate the integrated electron density along the line-of-sight.

By measuring rotation measures for the highest density of sources possible, and over a wide range of Galactic longitudes, one can thus accurately reconstruct the large-scale Galactic magnetic field in the disk. Much effort has been put into these studies, and experiments such as the Canadian Galactic Plane Survey (Taylor et al., 2003) and the Southern Galactic Plane Survey (McClure-Griffiths et al., 2005; Haverkorn et al., 2006) have now observed hundreds of square degrees near the Galactic plane with arcminute resolution. 


\subsubsection{Rotation Measure Synthesis}

Rotation measure synthesis, also called Faraday tomography, is a novel technique for 3-D mapping of RM structures in the ISM. The idea was described by Burn (1966) decades ago, but only now are spectropolarimetric capabilities advanced enough that we can use this technique (Brentjens \& de Bruyn, 2005). The Faraday depth of a rotating medium is defined as

$$
\phi \equiv 0.812 \int_{x_{1}}^{x_{2}} n_{\mathrm{e}} \mathbf{B} \cdot \mathrm{d} \mathbf{l}\left(\operatorname{rad~m}^{-2}\right)
$$

where the integral runs over a particular segment of the line-of-sight, from $x_{1}$ to $x_{2}$, and $n_{\mathrm{e}}$, $\mathbf{B}$, and $\mathrm{d} \mathbf{l}$ have the same meaning and units as in Sec. 2.1.2. In general, $\phi \neq \mathrm{RM}$. In addition, one can define the observed polarized flux density function as $\bar{P}\left(\lambda^{2}\right) \equiv W\left(\lambda^{2}\right) P\left(\lambda^{2}\right)$, where $P\left(\lambda^{2}\right)$ is the complex polarized surface brightness of the source as a function of wavelength, and $W\left(\lambda^{2}\right)$ is a sampling function which is non-zero only at the wavelengths where measurements are taken. Fourier transformation of the polarized surface brightness as a function of $\lambda^{2}$ gives $F(\phi)$, the complex polarized surface brightness per unit Faraday depth $\phi$ through

$$
F(\phi)=\frac{1}{\pi} \int_{-\infty}^{+\infty} P\left(\lambda^{2}\right) \mathrm{e}^{-2 \mathbf{i} \phi \lambda^{2}} \mathrm{~d}\left(\lambda^{2}\right)
$$

Thus, provided one can account for the effect of the sampling function, which is possible when many wavelengths are observed (Brentjens \& de Bruyn, 2005), and make reasonable assumptions as to the definition of $P$ for $\lambda^{2} \leq 0$ (Burn, 1966), RM synthesis provides a 3-D map of polarized intensity, where the third dimension is Faraday depth. However, Faraday depth does not necessarily increase monotonically with distance, as significant changes in field direction, electron density, or magnetic field strength will also impact its value, and there is therefore no direct distance information in these maps. Morphological correspondences with structures indicating a dramatic change in the magneto-ionic medium along the observed line-of-sight, such as spiral arms, supernova remnants, or external galaxies, can provide this missing link. Since the traditional way of measuring RMs only provides one RM value for each line-of-sight, and none where depolarization is severe, this new technique provides a tremendous increase in the amount of obtainable information from radio spectropolarimetric surveys.

\subsubsection{Other Techniques}

Polarized Dust Emission Non-spherical dust grains can partially align under the influence of an external magnetic field, which causes the emission from interstellar dust at far-infrared and submm wavelengths to be polarized. Although tremendous progress has been made in recent years (see, e.g., the review by Lazarian, 2007), the grain alignment mechanisms are not yet fully understood. As the degree of polarization of this emission depends not only on the magnetic field strength but also on dust properties and alignment mechanisms, observations of polarized emission from interstellar dust do not currently allow the magnetic field strength to be directly inferred. However, the magnetic field direction averaged over the line-of-sight can be measured, as the polarization angle is perpendicular to the component of the magnetic field in the plane of the sky. This method has so far been mostly used in 
denser regions of the Galaxy, such as molecular clouds and cores (see, e.g., Vaillancourt, 2007). In these environments, a rough estimate of the strength of the magnetic field can be obtained with the Chandrasekhar-Fermi method (Chandrasekhar \& Fermi, 1953) by comparing the dispersion in polarization angle to the turbulent velocity dispersion. Numerical simulations suggest that results from this technique should be corrected by factors of order unity (Heitsch et al., 2001).

Starlight Polarization When non-spherical dust grains are (partially) aligned with the Galactic magnetic field, the dusty region they are part of acts like a polarizer. As absorption of radiation by dust grains is more efficient in the direction of their long axes, initially unpolarized starlight illuminating the region becomes polarized in the direction of the grains' short axes, i.e., mostly parallel to the Galactic magnetic field (see Lazarian, 2007, for a more detailed discussion). By observing the polarization of the remaining starlight, inferences can therefore be made about the Galactic magnetic field required to produce the observed polarization close to the Sun, or, more generally, in regions of low dust extinction (see, e.g., Fosalba et al., 2002).

Zeeman Splitting In the presence of a magnetic field, degeneracies in atomic or molecular energy levels can be lifted. The energy separation between the previously degenerate Zeeman sub-levels is proportional to the strength of the magnetic field. As a result, a sufficiently large field must be present for the corresponding separation in a given radio-frequency line for a given species of atom or molecule to be detectable. In general, the magnitude of the interstellar magnetic field $(\sim 10 \mu \mathrm{G})$ is not strong enough to generate detectable Zeeman splitting (see, e.g., Davies et al., 1960) outside of localized interstellar clouds where the field is at least $\sim 50 \mu \mathrm{G}$ (see, among others, the review by Davies, 2007).

That being said, recent studies of Zeeman splitting from $\mathrm{OH}$ masers in massive star forming regions (Fish et al., 2003) and within molecular clouds (Han \& Zhang, 2007) have suggested that the fields within these regions are influenced by, and even reminiscent of, the large-scale Galactic field. While these results seem to be consistent with observations from other methods, more data is required before it can be considered a new way of constraining the Galactic field.

Although Zeeman splitting can provide information on the strength of the Galactic magnetic field, it does not encode information related to its direction. Observations of the Goldreich-Kylafis effect (Goldreich \& Kylafis, 1981), which leads to a small linear polarization of interstellar radio-frequency lines, could complement Zeeman constraints from molecular clouds and star forming regions by providing the direction of the sky-projected component of the field.

UHECR Multiplets The deflection angle of a ultrahigh energy cosmic ray (UHECR) is independent of the Galactic electron density, and is proportional to its charge $Z$ and the transverse magnetic field, while being inversely proportional to its energy $E$. Thus, UHECRs originating from the same source will have their arrival directions appear, roughly, as a string on the sky, with the cosmic rays with higher value of $Z / E$ further from the true 
source direction. By detecting these multiplets, one can therefore estimate the direction of the source and the strength of the integrated transverse magnetic field along the line-ofsight (Jansson et al., 2007). This offers a direct way of constraining models of the Galactic magnetic field without having to rely on a model for the Galactic electron density (on this topic, see also Sec. 3.3).

Although using this technique will have to wait until a bigger UHECR data set is available, it is worth noting that the Pierre Auger collaboration (Abraham et al., 2007), after 1.2 years of observations, found correlations between 28 cosmic ray events above 60 $\mathrm{EeV}$ and the positions of nearby AGNs. In ten years, over 200 events at that energy, including many multiplets, can therefore be expected in the Auger data, which could lead to potentially interesting constraints.

\subsection{Current Knowledge}

For practical reasons, the Galactic magnetic field is usually discussed as consisting of two components: the large-scale and the small-scale field. The large-scale, or "regular," magnetic field is predominantly azimuthal in the plane of the Milky Way, as clearly shown by the direction of starlight polarization by interstellar dust (Heiles, 2000) and confirmed with the lowest-frequency maps in the WMAP 3-year polarization data (Page et al., 2007). The small-scale, or "random," component is of comparable strength as the regular field, exhibits structure over a wide range of physical scales, and is presumably turbulent (see discussion in Sec. 2.2.2).

The average strength of the total magnetic field, i.e., regular and random components together, can be derived from observations of the Galactic synchrotron emission, as discussed in Sec. 2.1.1. Under the assumption of equipartition, the total magnetic field strength is estimated at $6 \pm 2 \mu \mathrm{G}$ in the solar neighborhood, and is found to increase to $10 \pm 3 \mu \mathrm{G}$ at a Galactocentric radius of $3 \mathrm{kpc}$ (Beck, 2001). The equipartition assumption used in these estimates has been validated by measurements of the local $\gamma$-ray density, which yields comparable field strengths as a function of radius (Strong et al., 2000). These results are consistent with those of Heiles (1996), who combined various methods to conclude that the total magnetic field strength is about $6 \mu \mathrm{G}$ in the spiral arms and $4 \mu \mathrm{G}$ elsewhere. Near the Galactic center, the magnetic field strength increases to several mG (Yusef-Zadeh et al., 1996).

In Sec. 2.2.1, we review the properties of the regular component of the Galactic field, whereas our knowledge of the random field is presented in Sec. 2.2.2. A Galactic halo field is also required to explain observations at low radio frequencies, which we discuss in Sec. 2.2.3.

\subsubsection{The Large-Scale Magnetic Field in the Galactic Disk}

The local regular field strength estimated from pulsar RMs combined with their dispersion measures (DMs) is about $1.4 \mu \mathrm{G}$ (see, e.g., Rand \& Lyne, 1994), while estimates from radio synchrotron intensity give higher values of $\sim 4 \mu \mathrm{G}$. Because of uncertainties and assumptions in both methods (Beck et al., 2003), these results are not necessarily in contradiction.

The large-scale magnetic field is observed to be concentrated in the Galactic disk (see, 
e.g., Simard-Normandin \& Kronberg, 1980; Han \& Qiao, 1994), and is generally assumed to be closely aligned with the Galactic spiral arms, consistent with observations of external galaxies (Beck, 2001). One feature that is critical in the determination of the dynamo mode(s) operating in the Galaxy is the presence of magnetic field reversals, regions of magnetic shear where the field direction changes by roughly $180^{\circ}$. One large-scale field reversal unquestionably exists between the local arm and the Sagittarius-Carina spiral arm of our galaxy, in the first quadrant (Simard-Normandin \& Kronberg, 1979; Thomson \& Nelson, 1980). There are indications that this reversal slices through the Sagittarius-Carina arm near $\ell=0$ (Weisberg et al., 2004; Brown et al., 2007), suggesting a significantly different inclination of the field from the spiral arms, at least in this region. The existence of additional reversals is highly controversial (see, e.g., Vallée, 2002; Weisberg et al., 2004; Han et al., 2006; Brown et al., 2007), and recent modeling based on RM data (Noutsos et al., 2008; Men et al., 2008), as well as on a combination of Faraday rotation and diffuse Galactic synchrotron emission measurements (see Sec. 3.3), all conclude that the current data is not sufficient to conclusively distinguish between several large-scale magnetic field models predicting differing numbers and locations of reversals. It is worth pointing out that magnetic field reversals have yet to be observed in external spiral galaxies (Beck, 2007). It is uncertain if this is a consequence of observation techniques and resolution, or a true indication of some uniqueness in the properties of the Galaxy.

Figure 1 illustrates the directions of the large-scale Galactic magnetic field superposed on the electron density model of Cordes \& Lazio (2002). Both universally accepted (solid arrows) and still debated (dashed arrows) directions are shown (see caption of Fig. 1 for more details).

\subsubsection{The Small-Scale Magnetic Field in the Galactic Disk}

The ratio of the regular to the small-scale Galactic magnetic field strength is estimated from synchrotron emission to be approximately 0.6 - 1 (see, e.g., Phillipps et al., 1981; Heiles, 1995), in agreement with estimates from the variance in pulsar RMs (Rand \& Kulkarni, 1989).

As the energy density in the magnetic field is comparable to the (turbulent) gas density (Boulares \& Cox, 1990), interstellar turbulence is expected to be magnetic, although details about its structure, strength and variability are still largely unknown. Interstellar turbulence is traditionally measured through power spectra of either velocity fluctuations in emission or absorption lines, or density variations. Similarly, one can get an idea of the role of the magnetic field by studying power spectra of RM measurements. To avoid the problem of depolarization along the line-of-sight, this is usually done using RMs of pulsars and polarized extragalactic sources. The corresponding data points being irregularly spaced, the computation of a power spectrum is usually replaced by a structure function analysis, with the second order structure function of RM given by $D_{\mathrm{RM}}(\mathrm{d} x)=\langle\operatorname{RM}(x+\mathrm{d} x)-\operatorname{RM}(x)\rangle_{x}$, where $\mathrm{d} x$ is the distance between two sources, and $\langle\cdot\rangle_{x}$ denotes averaging over all positions $x$. These analyses show that RM fluctuations exist over a wide range of scales and exhibit a power law behavior (see, e.g., Minter \& Spangler, 1996). The largest observed fluctuations are on scales of $50-100$ pc when averaged over the sky (Rand \& Kulkarni, 1989; Ohno 
\& Shibata, 1993), and of order 1 pe in the spiral arms (Haverkorn et al., 2008). At least part of this structure is caused by magnetic field fluctuations as opposed to variations in the electron density (Minter \& Spangler, 1996).



Figure 1: Directions of the large-scale Galactic magnetic field as viewed from the North Galactic pole. The grey scale represents the electron density model of Cordes \& Lazio (2002), Q1-4 indicate the four Galactic quadrants, and the asterisk shows the location of the Sun. Solid arrows correspond to universally accepted field directions, while single-ended dashed arrows show field directions as supported by the Canadian or the Southern Galactic Plane Survey (CGPS and SGPS, respectively), though not necessarily universally accepted (Brown et al., 2003b, 2007). Finally, double-ended arrows indicate regions remaining highly debated with either no data or no clear indications currently available from the CGPS or the SGPS.

\subsubsection{The Magnetic Field in the Galactic Halo}

Significant synchrotron emission is observed at high Galactic latitudes, indicating the presence of a magnetic field in the Galactic thick disk or halo. The regular magnetic field has a scale height of about $1.5 \mathrm{kpc}$ and is an order of magnitude weaker than the disk field, as derived from pulsar RMs (Han \& Qiao, 1994) and supported by extra-Galactic RMs (Brown et al., in preparation). In the thin disk of the Galaxy, the field points in the same direction above and below the Galactic plane (Frick et al., 2001). However, there is evidence for large-scale reversals of the field's direction in the thick disk/halo, which are likely caused either by a dipole structure of the halo magnetic field (Andreasyan \& Makarov, 1988; Han et al., 1997), or by local structures that appear large on the plane of the sky. 


\section{Global Constraints on the Magnetized ISM}

As reviewed in Sec. 2.1, numerous techniques can be used to probe the structure of the Galactic magnetic field on various scales. However, most of these studies do not lead to direct constraints on the latter, but on the magnetized interstellar medium, of which the Galactic magnetic field is only one component along with, e.g., thermal and cosmic ray electrons. Given a model for the physical properties of these other components, each technique can then be used to characterize the field in our galaxy. These models are usually themselves derived from other observations, and their reliability is often limited, which, in turn, weakens the constraints one can infer for the Galactic magnetic field. An example can be found in the controversy surrounding the existence of field reversals in the outer parts of the Galaxy (see Sec. 2.2.1).

Although this approach has several advantages, in particular insofar as it offers the possibility of comparing independent constraints on the various components of the field (see, e.g., Crutcher et al., 2003), it fails to account for the possible degeneracies between properties of the field and characteristics of other components of the magnetized interstellar medium. This can be avoided by simultaneously fitting multiple observations, which also has the advantage of leading to a unique model able to describe a diverse set of data. Such a model is all the more desirable since the same physical quantities are involved in various probes of the field.

There are multiple data sets one can combine to build such a model, but one first needs a simulation code able to generate the corresponding theoretical maps from a set of parameters describing the relevant components of the magnetized interstellar medium. The Hammurabi software (Waelkens et al., 2008) was developed to provide such a tool for the Galactic synchrotron emission (total and polarized), free-free emission, and the effect of Galaxy-induced Faraday rotation on the emission from extra-galactic sources. In this section, we report on the efforts by two groups: Jansson et al. (2007) and Sun et al. (2008). The former used HammURABI to constrain the magnetized interstellar medium with the three-year WMAP $23 \mathrm{GHz}$ polarization data (Page et al., 2007). Sun et al. (2008) then performed an extensive analysis taking into account the total synchrotron emission observed by Haslam et al. (1981, 1982), the $1.4 \mathrm{GHz}$ synchrotron map of Wolleben et al. (2006), the WMAP three-year free-free emission template (Hinshaw et al., 2007), and the rotation measures of Brown et al. (2003b, 2007).

\subsection{ISM Modeling}

Assumptions about three components of the magnetized ISM, namely, thermal electrons (Sec. 3.1.1), cosmic ray electrons (Sec. 3.1.2), and the Galactic magnetic field (Sec. 3.1.3), are necessary to make it possible for HAMMURABI to generate the simulated maps mentioned in Sec. 3. Comparison to data then enables Jansson et al. (2007) and Sun et al. (2008) to constrain the parameters of these models by making sure that they can reproduce their selected sets of observations. We review each of these models and their associated parameters below. Some of the constraints obtained by the Jansson and Sun groups are 
reviewed in Sec. 3.3 .

\subsubsection{Thermal Electron Density and Temperature}

HAmmuRABI uses the NE2001 model developed by Cordes \& Lazio $(2002,2003)$ to describe the Galactic thermal electron density $n_{\mathrm{e}}$. This model, based on dispersion measures, contains both a thick disk and a thin disk, as well as spiral arms and some local ISM features.

Sun et al. (2008) combined the results of Reynolds et al. (1999), Peterson \& Webber (2002), and Quireza et al. (2006) to show that the temperature distribution of the thermal electrons is well described by $T_{\mathrm{e}}(\mathbf{r}) / \mathrm{K}=5780+287 r-526|z|+1770 z^{2}$, where $r$ and $z$ (cylindrical coordinates) are in units of kpc. Moreover, the free-free emission intensity is proportional to $\left\langle n_{\mathrm{e}}{ }^{2}\right\rangle$, where the average is along a given line-of-sight, which is a priori different from the quantity $\left\langle n_{\mathrm{e}}\right\rangle^{2}$ that can be inferred from the NE2001 model. This discrepancy can be accounted for by the filling factor $f_{\mathrm{e}}(z) \equiv\left\langle n_{\mathrm{e}}\right\rangle^{2} /\left\langle n_{\mathrm{e}}{ }^{2}\right\rangle$ provided by Berkhuijsen et al. (2006).

\subsubsection{Cosmic Ray Electron Density}

Models of the cosmic ray electron density such as those used by Page et al. (2007) and Sun et al. (2008) are composed of a simple spatial distribution characterized by a scale height and a scale length, and also assume a power law energy distribution for the electrons. The latter has so far been sufficient to model foregrounds in the context of CMB data analysis [see, e.g., Tegmark \& Efstathiou (1996), de Oliveira-Costa et al. (2008), Dunkley et al. (2008b), and Bottino et al. (2008)]. However, simulation codes, such as GALPRoP, lead to models much closer to the state-of-the-art in cosmic ray modeling (as in Strong et al., 2007) and might have to be used in conjunction with more sophisticated analyses in the future (we refer the reader to the companion paper by Dunkley et al. (2008a) for details on this issue).

The Sun et al. (2008) model assumes a scale height (respectively, a radial scale length) of $1 \mathrm{kpc}$ (respectively, $8 \mathrm{kpc}$ ), whereas the normalization is chosen to reproduce observations at $\mathbf{r}=\mathbf{r}_{\odot}$ (see, e.g., Strong et al., 2007). Furthermore, a truncation is introduced for $|z|>1 \mathrm{kpc}$ to explain the low synchrotron emission observed at high Galactic latitudes. Finally, the energy distribution is given by the power law $N(E) \propto E^{-p}$, where $p$ is 3 for $\nu>408 \mathrm{MHz}$, and 2 at lower frequencies. No local features of the Galactic interstellar medium are modeled.

\subsubsection{Galactic Magnetic Field}

Any large-scale magnetic field model can be tested with HammuraBi. Jansson et al. (2007) looked at axisymmetric and bisymmetric spiral models, with symmetry or antisymmetry in the $z$ direction, while Sun et al. (2008), after demonstrating the need to improve models found in the literature, considered an axisymmetric spiral field with a ring, an axisymmetric spiral field with a field reversed arm, and a bisymmetric spiral field. In addition, a halo magnetic field, necessary to fit high-latitude rotation measure profiles, and a random field, 
required by the synchrotron and RM observations, are included in the Sun et al. (2008) analysis.

As for the cosmic ray electron model, no attempt has been made at modeling the Galactic magnetic field to explain local features such as the North Polar Spur. Although they occupy a large fraction of the sky, and are fascinating objects to explore on their own (see, e.g., the Loop I model developed by Wolleben, 2007), they are beyond the scope of a first global description of the diffuse Galactic emission, and are therefore not included in HAmmuraBI.

\subsection{Simulation Outputs}

Based on the model described in Sec. 3.1, Hammurabi can generate Galactic simulated maps of synchrotron emission (total and polarized), free-free emission, and rotation measure (Fig. 2). Structures like the North Polar Spur, or any of the other Loops, are not reproduced by these simulations since the magnetic field and cosmic ray models only reproduce the largescale features of the Galaxy. However, local features in the electron density distribution, which are part of the NE2001 model (see Sec. 3.1.1), can be recognized in the rotation measure map.
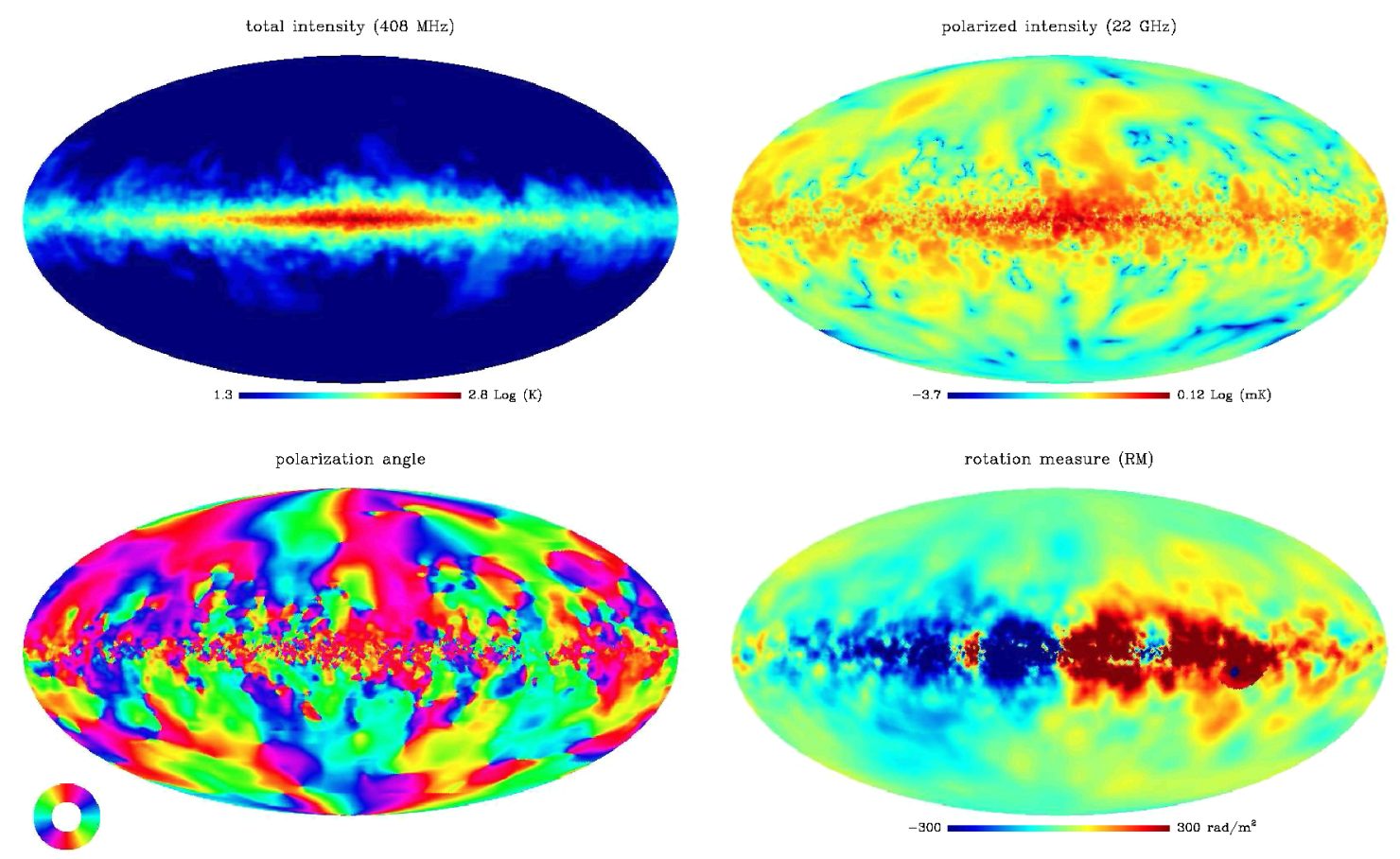

Figure 2: Mock observations of the Sun et al. (2008) model. The top row shows full-sky maps of the total (left) and polarized (right) synchrotron emission, whereas the bottom row provides a map of the corresponding polarization angle (left) and a rotation measure map (right). Unlike in Sun et al. (2008), the small-scale magnetic field is modeled as a Gaussian random field. The simulated map of free-free emission is not shown here. 


\subsection{Comparison to Observations}

Using a $\chi^{2}$ minimization technique, Jansson et al. (2007) compare the polarized synchrotron maps produced by HAMMURABI to the three-year WMAP K-band (23 GHz) map smoothed to $4^{\circ}$. They find that the minimized $\chi^{2}$ is close to identical for all models of the Galactic magnetic field they consider (see Sec. 3.1.3). However, for each of them, large regions of the parameter space can be excluded, even though there are no obvious best-fit parameters as a number of combinations yield a value of $\chi^{2}$ very close to the minimum. In particular, the vertical and radial scale-lengths can take unphysically large values, which is explained by the drop in synchrotron emission due to the reduction in the relativistic electron density at large radial and vertical distances. Thus, knowing the polarized synchrotron emission is not sufficient to strongly constrain the parameters of these Galactic magnetic field models.

By combining synchrotron emission data (Page et al., 2007) with rotation measure information (Brown et al., 2003b, 2007) and knowledge of the full-sky free-free emission (Hinshaw et al., 2007), Sun et al. (2008) showed that this degeneracy can be at least partially lifted. They indeed demonstrated that increasing the vertical scale-length of the NE2001 thermal electron density, removing the unphysical cut in the cosmic ray electron density (see Sec. 3.1.2), and decreasing the strength of the halo magnetic field, leads to a better global fit to the observations. The need for a thermal electron scale height about twice as large as that of the NE2001 model was confirmed by an independent data analysis by Gaensler et al. (2008).

Sun et al. (2008) were also able to find statistical evidence that the Galactic magnetic field is best fit by an axisymmetric spiral field with a ring located in the inner part of the Galaxy, thereby adding to the case that combining observations probing a broad range of physical processes is a powerful way of constraining the various components of the magnetized ISM.

\subsection{Future Developments}

In addition to the probes combined by Sun et al. (2008), other observations could already be used to improve our knowledge of the magnetized interstellar medium. An example is pulsar RM - DM plots (see, e.g., Brown et al., 2003b, and Sec. 2.2.1), which trace the three-dimensional structure of the Galactic magnetic field. HammURABI's design allows it to perform the corresponding mock observations, and the Square Kilometer Array (SKA) is expected to provide thousands of data points for these plots by 2020 (Beck \& Gaensler, 2004).

The Sun et al. (2008) model does not contain any dust related inputs, and, as a result, cannot make starlight polarization or dust emission predictions. Dust emission mechanisms are difficult to model given the sophisticated and sometimes unknown physics associated with them. Moreover, models developed to match all available observations can lead to significantly different predictions for what the polarized emission from interstellar dust might look like (see Sec. 5.3). Adding a dust component to HAmmuraBi will therefore be a challenging task.

Upcoming radio telescopes, such as the SKA, the LOw Frequency ARray (LOFAR), and 
the Atacama Large Millimeter Array (ALMA), associated to upcoming CMB experiments, such as Planck, will greatly increase the number and the quality of available observations. A tool like Hammurabi will allow us to make the most of these improvements, and, in particular, to generate valuable information regarding the structure and properties of the magnetized interstellar medium. This will be a welcomed source of inputs for the CMBPol data analysis.

\section{The Galactic Magnetic Field in the CMBPol Era}

\subsection{Radio Surveys in the Next Decade}

Narrow frequency and spatial coverage have so far been the main limiting factors in studying the low frequency polarized emission from the Galaxy. This issue is expected to be addressed by large radio polarimetric surveys currently planned or underway, which will provide a wealth of new data by the time CMBPol flies. The S-PASS polarimetric survey (PI: E. Carretti) will observe the entire Southern sky in a $256 \mathrm{MHz}$ frequency band centered on $2.3 \mathrm{GHz}$ by 2009. The GALFACTS polarized continuum survey (PI: A. R. Taylor) will map the entire sky visible from Arecibo in a $300 \mathrm{MHz}$ frequency band centered on $1.4 \mathrm{GHz}$ by 2012, whereas the STAPS survey (PI: M. Haverkorn) will cover $500 \mathrm{MHz}$ around the same frequency in the Southern sky. Radio polarimetric observations are also planned with the Australian SKA Pathfinder (Johnston et al., 2007), which should be fully operational by 2013. Finally, the Galactic Magneto-Ionic Medium Survey (GMIMS, PI: M. Wolleben), a major project consisting of six independent radio polarimetric surveys, is collecting highresolution $\left(\sim 30^{\prime}\right)$ all-sky polarization data in a near-continuous frequency band ranging from $300 \mathrm{MHz}$ to $1.8 \mathrm{GHz}$.

These and similar surveys will provide "RM grids" (Beck \& Gaensler, 2004) gathering numerous measurements of the Galactic Faraday rotation of polarized emission from extragalactic sources across the whole sky. Although EGSs have a small intrinsic RM component, it can be statistically eliminated by averaging the RMs of enough point sources in a given patch of the sky. The current average RM source density is about 1 RM source per 10 square degrees at high latitudes, and approximately 1 source per square degree in parts of the Galactic plane (Brown et al., 2003a, 2007). As a result, inferred Galactic RM maps are very low resolution over most of the sky. However, the radio surveys described above are expected to fill in the Galactic plane to the level of 1 source per square degree, as well as to increase the source density at high latitudes by a factor of 100 . This will allow averaging of a sufficient number of RM sources to remove intrinsic RM contributions on scales of about $1^{\circ}$. Detailed modeling of the pitch angle of the Galactic magnetic field, the number and locations of large-scale field reversals, and the vertical component of the magnetic field will therefore soon become possible.

Moreover, RM grids will enable detailed study of the turbulent field component over a wide range of scales and large parts of the sky. Given their expected high source density, it will become possible to construct a structure function every few square degrees on the sky, which will result in unprecedented detailed knowledge of the turbulent behavior of the magnetized ISM on degree scales. In particular, possible changes in the turbulence 
characteristics from the Galactic plane, via the disk-halo connection to the Galactic halo, should become visible.

Finally, RM synthesis of polarized emission from diffuse synchrotron emitting gas in the Galaxy can be used to obtain a 3-dimensional map of Galactic RM structure. All of the above mentioned surveys have the capability to perform RM synthesis on their data, with various limitations, which will be used to improve modeling of the large-scale components of the Galactic magnetic field. As the technique allows separation of magnetic foregrounds and backgrounds, it will also be possible to study magnetic field structures in Galactic objects such as supernova remnants, HII regions, and planetary nebulae, as well as in external galaxies.

\subsection{The Contribution of Upcoming CMB Experiments}

To study the global character of the Galactic magnetic field through synchrotron emission and (de)polarization, observations over a significant part of the sky are needed, at a frequency high enough that Faraday rotation is negligible. WMAP provides polarization maps that allow study of the Galactic magnetic field through synchrotron radiation (Page et al., 2007). However, the polarized emission in the lowest frequency WMAP maps has a signal to noise ratio below 3 in about $55 \%$ of the sky when smoothed to $2^{\circ}$. The only polarized structures that are clearly visible in the WMAP maps are the Galactic plane, the region of high and regular polarization called the Fan region (Berkhuijsen et al., 1964; Brouw \& Spoelstra, 1976), and the North Polar Spur (Hanbury Brown et al., 1960), generally believed to be an old, nearby supernova remnant. Therefore, to be able to study the behavior of the synchrotron radiation away from the Galactic plane in the "typical" interstellar medium, higher sensitivity observations are needed. WMAP polarization maps show that although in both the Galactic plane and discrete structures the polarized intensity can go up to $\sim 100 \mu \mathrm{K}$, at intermediate and high latitudes the polarized intensity is primarily below $20 \mu \mathrm{K}$. This will allow a nominal detection with Planck or nine years of WMAP observations, but with a typical signal-to-noise lower than 2 , which will lead to an error in polarization angle determination of at least $1 \mathrm{rad}$. CMBPol, which could be 10 times more sensitive than Planck at the same frequencies, would have much higher signal-to-noise across the whole sky, but in particular in these intermediate and high Galactic latitude regions. This data would complement the information provided by the radio surveys described in Sec. 4.1, in particular with respect to the vertical component of the field, as well as the strength and structure of the halo field, both of which are necessary knowledge to test dynamo theories of the origin and evolution of Galactic magnetism.

In addition, the resolution of CMBPol might be higher than that of Planck, which would significantly decrease depolarization. At the frequencies currently considered for CMBPol, there is no significant Faraday depolarization, but small-scale structure in the magnetic field will cause small-scale structure in polarization angles of the synchrotron emission, resulting in depolarization of the radiation within one telescope beam. If the satellite's resolution is not high enough, the polarized emission will be below its detection threshold, destroying all diagnostic capability of the data for Galactic magnetic fields. However, at high latitudes, turbulent cells are expected to be at $\sim 100$ pe scales (Dumke et al., 1995), 
which, at the distances of interest, corresponds to a much larger angle than the resolution currently considered for CMBPol. As a result, depolarization is not expected to be severe there. On the other hand, the typical structure of the magneto-ionized medium in spiral arms could be on scales as small as 1 pc (Haverkorn et al., 2008), which makes depolarization resolution dependent. In particular, higher resolution than Planck's $33^{\prime}$ beam at $30 \mathrm{GHz}$ will be necessary to observe turbulent structures in the magneto-ionized medium in and close to the Galactic plane.

The same holds for discrete polarized structures, such as supernova remnants (SNRs). Statistical studies of supernova rates (see, e.g., Li et al., 1991) indicate that there should be many more SNRs in the Galaxy than are currently known. This "missing SNRs" problem is likely due to a selection effect of many radio surveys against large low-brightness SNRs as well as small remnants. However, low-brightness remnants might be observable through their magnetic field structure, which remains visible through polarized radio emission even when synchrotron intensities are too low to be observed (Haverkorn, 2005). A sensitive all-sky survey like CMBPol with the same resolution as necessary to study the turbulent structure of the field in spiral arms therefore opens the possibility of detecting the magnetic

field structures of old and faint SNRs, which could help solve the long-standing "missing SNRs" problem.

\section{Polarized Dust Emission (PDE)}

Over the course of the last 60 years, the linear polarization of starlight has been observed at wavelengths ranging from the ultraviolet (Clayton et al., 1992; Anderson et al., 1996; Martin et al., 1999) to the far-infrared (see, e.g., the review by Whittet, 2003) along numerous linesof-sight. The Archeops experiment first released detailed studies of the emission properties of dust at CMB wavelengths based on observations of $20 \%$ of the sky at $353 \mathrm{GHz}$ (Benoît et al., 2004; Ponthieu et al., 2005), and warned that the polarized dust emission would be a major contaminant of the primordial E- and B-mode signals unless removed properly. These results were largely confirmed by the WMAP mission (Kogut et al., 2007), although the fact that the highest WMAP frequency $(94 \mathrm{GHz})$ is below $100 \mathrm{GHz}$ made it easier to handle this emission in the polarization analysis (Page et al., 2007; Gold et al., 2008).

The Planck satellite will soon be on its way to L2, where it will map the sky at frequencies ranging from $30 \mathrm{GHz}$ to $857 \mathrm{GHz}$, with 5 of its bands covering frequencies higher than $100 \mathrm{GHz}$. Compared to WMAP, Planck will have twice as many frequency channels, 3 times higher resolution, and 10 times higher sensitivity, all of which will, by design, be of tremendous help to distinguish between polarized foregrounds and candidate inflationary signals.

However, a number of assumptions had to be made to reach this "optimal" configuration. In particular, the dust emission, which will be brighter in the 5 Planck frequency bands mentioned above than in any WMAP map, was assumed to have the same polarization degree at all wavelengths, with the $857 \mathrm{GHz}$ observations providing a way of determining, among other information, this polarized fraction. Although so far consistent with the degrees of polarization inferred by the Archeops and WMAP groups (Benoit et al., 2004; 
Kogut et al., 2007) at, respectively, $353 \mathrm{GHz}$ and $94 \mathrm{GHz}$, there is no strong observational evidence that this will hold at all Planck frequencies. If the level of polarized dust emission varies as a function of frequency, it will reduce our ability to remove this emission from the Planck maps. A CMBPol mission is likely to have between 4 and 6 frequency bands in which the brightest signal will be dust emission, and might therefore have to deal with the same problem.

In this section, we follow Draine \& Fraisse (2008), and show how our current knowledge of the extinction and polarization of starlight can be used to predict the degree of polarized dust emission one could observe at CMB wavelengths with a high-resolution and high-sensitivity mission such as Planck or CMBPol, as well as its potential dependence on frequency.

\subsection{Observed Extinction and Polarization of Starlight}

Although observations of the extinction and polarization of starlight exist at numerous wavelengths [see references in the first paragraph of Sec. 5, as well as Martin et al. (1992), and, e.g., the review by Fitzpatrick (2004)], they are well fit by simple parametric functions. Fitting the average Galactic extinction curve is discussed in Fitzpatrick (1999) for $0.1<$ $\lambda / \mu \mathrm{m}<2.65$, whereas Anderson et al. (1996) showed that the fractional polarization curve $p(\lambda)$ is well described by a Serkowski law (Serkowski, 1973) for $0.15<\lambda / \mu \mathrm{m}<1.39$. Finally, for $1.39<\lambda / \mu \mathrm{m}<5, p(\lambda) \propto \lambda^{-1.7}$ is a good match to the observations by Martin et al. (1992). It is therefore possible to represent the observed extinction and polarization of starlight by smooth continuous functions in the range of wavelengths for which data is available.

The fact that we observe starlight polarization implies that dust grains cannot be spherical, and that they have to be at least partially aligned with the Galactic magnetic field. However, the data currently in hand does not provide direct constraints on the shape of grains, their size distribution(s), or their alignment function(s). As a result, modeling interstellar dust requires relying on theory to predict the general behavior expected for the latter. One can then assume a shape and try to fit the observed extinction and polarization of starlight. Of course, this process does not guarantee that there is a unique good fit as only a few shapes can be tested in a reasonable amount of time. But the hope is that, given the many constraints imposed on candidate models, if several of them can accurately reproduce observations of the extinction and polarization of starlight over a wide range of wavelengths, they will lead to similar predictions for what the polarized dust emission should look like at CMB wavelengths, at least as long as the non-spherical interstellar grains are made of the same material(s).

\subsection{Modeling Interstellar Dust}

Dust grains are believed to be made of the numerous elements that have been depleted from the gas phase (Jenkins, 2004). However, observed spectroscopic features in the Galactic extinction curve and in observations of emission by interstellar dust in the mid-infrared indicate that the bulk of the mass in interstellar grains is in the form of amorphous silicates 
and carbonaceous materials, among which a population of Polycyclic Aromatic Hydrocarbons (PAHs) is required to explain several emission features (see, e.g., the review by Draine, 2003).

In order to explain the polarization observed in some of the spectroscopic features that have been studied in the far-infrared, amorphous silicates have to be present in non-spherical dust grains aligned with the Galactic magnetic field, B (see, e.g., Whittet, 2003). As of today, there is however no compelling evidence that the same is true for carbonaceous materials (Chiar et al., 2006). Finally, PAHs are expected to have their angular momenta only slightly aligned with B, and a PAH's principal axis of largest moment of inertia will be only partially aligned with its angular momentum (Lazarian \& Draine, 2000). Furthermore, PAHs are thought to contribute only a small fraction of the total emission by dust at frequencies $\nu \lesssim 10^{3} \mathrm{GHz}$. As a result, PAHs should contribute negligibly to the polarized emission at those frequencies.

\subsection{Far-Infrared Emission from Interstellar Dust}

Based on the considerations discussed in Sec. 5.2, Draine \& Fraisse (2008) fit the observed extinction and polarization of starlight with dust models consisting of (1) a population of (nearly) randomly-oriented PAHs, (2) oblate spheroidal silicate grains, and (3) graphite grains assumed to be either spheres (in which case their emission is not polarized) or oblate spheroids with the same axial ratio $b / a$ of their equatorial to their polar axes as the silicates.

Table 2: Draine \& Fraisse (2008) Dust Models

\begin{tabular}{ccc}
\hline \hline Model number & $(b / a)_{\text {silicate }}$ & $(b / a)_{\text {carbon }}$ \\
\hline 1 & 1.4 & 1.0 \\
2 & 1.4 & 1.4 \\
3 & 1.6 & 1.0 \\
4 & 1.6 & 1.6 \\
\hline \hline
\end{tabular}

Table 2 provides the values of this ratio for each component of the Draine \& Fraisse (2008) dust models, while Fig. 3 shows the corresponding predicted far-infrared emission spectra, as well as its expected degree of polarization as a function of wavelength, under the assumption that all dust grains are heated by the Mathis et al. (1983) Galactic radiation field, and that the observer is looking in a direction perpendicular to the Galactic magnetic field.

Despite the wide range of axial ratios probed by the 4 models listed in Tab. 2 for both amorphous silicate and carbonaceous grains, Draine \& Fraisse (2008) showed that they all provide adequate fits (not shown here) to the observed extinction and polarization of starlight, and give far-infrared emissions compatible with the DIRBE measurements (see Fig. 3). The emission predicted at CMB wavelengths is also remarkably similar between all models. 



Figure 3: (Left) Far-infrared emission spectra and (Right) corresponding degrees of polarization calculated by Draine \& Fraisse (2008) for the models in Tab. 2. On each plot, the vertical dotted lines indicate 4 possible CMBPol frequencies ranging from $100 \mathrm{GHz}$ to $850 \mathrm{GHz}$. The symbols on the emission spectra represent the $\mathrm{HI}$ correlated emission as measured by DIRBE at high Galactic latitudes (Dwek et al., 1997; Arendt et al., 1998).

However, the expected degree of polarization of the far-infrared emission from interstellar dust and its wavelength dependence are strong functions of the class of models one considers. When both silicate and carbonaceous grains are non-spherical and partially aligned with the Galactic magnetic field, the fractional polarization of the dust emission is essentially independent of frequency at CMB wavelengths, and of order $10 \%$. But if only the emission by silicates contributes to the polarized signal, the polarized fraction of the dust emission can vary by a factor of 1.4 between 850 and $100 \mathrm{GHz}$, from around $11 \%$ to over $15 \%$. For a given class of models, the level of polarization calculated by Draine \& Fraisse (2008) is not very sensitive to the value of the ratio $b / a$ considered, which makes their predictions relatively robust.

\subsection{Polarized Fraction of Dust Emission for CMB Forecasts}

The polarized fractions shown in Fig. 3 may seem high in light of the degrees of polarization reported by Archeops (Benoît et al., 2004) or WMAP (Kogut et al., 2007) for the diffuse Galactic emission. However, there should be very few lines-of-sight in the sky close to perpendicular to the Galactic magnetic field, so the average numbers these groups report, ranging from $4 \%$ to $7 \%$, should indeed be smaller. That said, on a given sightline, the ratio of the observed polarization to the polarization calculated for any of the models in Tab. 2 should not be a function of frequency. Therefore, if one believes that the Galactic magnetic field models used by Archeops and WMAP are accurate enough to get a good estimate of the polarized fraction of dust emission of $5 \%$ at $100 \mathrm{GHz}$, we can easily rescale the Draine 
\& Fraisse (2008) models to reproduce this observation. All of them are then consistent with a polarized fraction between $3 \%$ and $5 \%$ at all frequencies CMBPol would potentially observe.

Depending on the precision to which the polarized fraction $\Pi$ of dust emission needs to be determined in order to achieve a mission's science goals, such as detecting B-modes, several scenarios might therefore have to be considered in a CMB forecasting pipeline:

- $\Pi=x \%$ and is independent of frequency for $\nu \lesssim 900 \mathrm{GHz}$ [as is approximately the case for models 2 and 4 of Draine \& Fraisse (2008)],

- $\Pi$ is a decreasing function of frequency, smoothly varying from $\Pi=x \%$ at $\sim 100 \mathrm{GHz}$ to $\sim 0.7 x \%$ at $\sim 850 \mathrm{GHz}$ [as in models 1 and 3 of Draine \& Fraisse (2008)],

where $x$ is chosen either as having a value corresponding to one of the models shown in Fig. 3, or from an estimate obtained by the Archeops or the WMAP groups. In the former case, one should keep in mind that the chosen value will likely be an overestimate of the observable degree of polarization, whereas in the latter case, the polarized fraction might be underestimated compared to its true value due to the coarse resolution and low sensitivity of the Archeops and WMAP instruments compared to the Planck or the CMBPol detectors.

\subsection{Constraining Dust Models with CMBPol}

With its many channels and its high resolution, high sensitivity detectors, CMBPol should be able to average the polarized fraction of dust emission on much smaller patches than WMAP (Kogut et al., 2007), with potentially higher precision, and to detect variations of the degree of polarization between frequency channels if it exists, and if the polarized fraction of dust emission is high enough. How well this can be achieved will of course depend on various factors, including the quality of the Galactic magnetic field models that we will be able to put together by the time the CMBPol data is analyzed. As a result, it is hard, if not impossible, to know what CMBPol will bring to the debate on what interstellar dust grains look like, and how they behave. However, we can envision two main game-changing scenarios:

- Consistent observations of low polarized fractions of dust emission (typically, $\lesssim 5 \%$ ) at all CMB frequencies would suggest that the Draine \& Fraisse (2008) models in which only silicate grains contribute to the polarized emission are not an obvious good match to the measurements. In this event, we might have to conclude that carbonaceous grains are significantly non-spherical and somewhat aligned with the Galactic magnetic field (as in their models 2 and 4), a fact for which we have currently no evidence (see Chiar et al. (2006) and discussion in Sec. 5.2). Alternatively, it could mean that the adopted opacities of the silicate material have to be reduced (in a wavelength-dependent fashion), while those of the carbonaceous grains should be correspondingly increased.

- However, if the polarized fraction of dust emission is found to be significantly decreasing when frequency increases, and potentially higher than what the Archeops 
and WMAP results suggest, this would be another indication that amorphous silicate grains and carbonaceous grains indeed form separate populations, with the polarized

emission being dominated by the former. This would pose a challenge to theories of grain alignment.

We also have to mention the possibility that a behavior completely different from the results shown in Fig. 3 could be observed. Although the models of Draine \& Fraisse (2008) appear to be consistent with all current observations, our understanding of interstellar dust is far from perfect. Such observations would therefore likely lead us to revisit some of the assumptions of our models for interstellar dust, opening a new window on dust properties and dust physics.

\subsection{Tracing PDE with Starlight Polarization}

The Draine \& Fraisse (2008) results predict the polarized fraction of far-infrared and submillimeter dust emission, and its dependence with wavelength, for lines-of-sight perpendicular to the Galactic magnetic field. Going from observed degrees of polarization to a function directly useable to constrain dust models (see Sec. 5.5), and, in particular, theories of grain alignment (see Sec. 6.3), therefore requires as good a large-scale magnetic field template as possible.

As mentioned in Sec. 2.1.4, optical or near-infrared starlight polarization gives us the sky-projected direction of the Galactic magnetic field integrated along lines-of-sight going through regions of low dust extinction (typically, $A_{V}$ or $A_{K} \lesssim 5$ mag). As a result, not much can be learned from this technique when it comes to studying the large-scale magnetic field in the Galactic disk, since it cannot probe the field in or near the Plane outside of the solar neighborhood. However, at intermediate and high Galactic latitudes, $|b| \gtrsim 10^{\circ}$, dust extinction is relatively low (see, e.g., Burstein \& Heiles, 1982, and references therein), with $A_{V} \simeq 0.03$ mag near the Galactic poles (the estimated error on this number is of order the extinction itself). Since these latitudes correspond to the regions of main interest to the CMB community, useful magnetic field templates can therefore be constructed from starlight polarization observations.

Heiles (2000) compiled a catalog, the largest to date, of the polarization of starlight from nearly 9300 stars. However, among those, Fosalba et al. (2002) only found $\sim 1400$ stars with reliable distance and extinction estimates lying sufficiently far from the Galactic plane, i.e., with $|b|>10^{\circ}$, to be useful for CMB purposes. Moreover, the vast majority of these stars, about 1300 , are less than $1 \mathrm{kpc}$ away from the Sun. Despite this lack of information outside of the plane, starlight polarization data has already been useful to CMB polarization studies. Page et al. (2007) and Dunkley et al. (2008b) both used the Heiles (2000) catalog to inform their fit to the dust emission component of their signal, and Kogut et al. (2007) showed that using the polarization angle provided by starlight polarization observations leads to a (marginally) better fit to this component than a model based on WMAP's low frequency polarized synchrotron observations. Since both starlight polarization and polarized dust emission are processes closely related to how grains align with the interstellar magnetic field and to the optical properties of interstellar dust grains, 
this improvement is not totally unexpected.

The scarcity of optical and near-infrared starlight polarization data has led several groups to try and remedy the situation, especially in view of the accuracies now achievable (see, e.g., Carciofi et al., 2007). An example is the Galactic Plane Infrared Polarization Survey (GPIPS), which aims at measuring nearly half a million $H$-band stellar polarizations for $H$ down to $12 \mathrm{mag}$ across a 72 square degree region of the inner Northern Galactic plane by 2011 (Clemens et al., 2007). But neither the GPIPS nor other surveys currently planed or underway (see, e.g., Magalhães et al., 2005; Nishiyama et al., 2008) will lead to a significant increase in the number of available data points in the diffuse interstellar medium at high Galactic latitudes. Given the implications this new data would have for our understanding of dust processes, but also for our ability to separate polarized dust emission from a potential gravity wave signal, we believe that acquiring it should be a high priority goal achieved by the time CMBPol flies.

\section{Constraining Grain Alignment Mechanisms}

The mechanism explaining how non-spherical dust grains align with a magnetic field has been sought for ever since Hall (1949) and Hiltner (1949) discovered dust-induced starlight extinction polarization. This quest has been highly controversial, and numerous mechanisms have been proposed and developed to various degrees over the years (see, e.g., the review by Lazarian, 2007). Among those is the paramagnetic Davis-Greenstein mechanism that has been matured through intensive work (Jones \& Spitzer, 1967; Purcell, 1979; Spitzer \& McGlynn, 1979; Mathis, 1986; Roberge et al., 1993; Lazarian, 1997; Roberge \& Lazarian, 1999) since its introduction by Davis \& Greenstein (1951). However, this "textbook solution" to the grain alignment problem has several shortcomings, among which the fact that thermal flipping prevents grains with effective radii $a \lesssim 1 \mu \mathrm{m}$ from aligning (Lazarian, 2007), which is hard to reconcile with fits to starlight polarization data (Draine \& Fraisse, 2008). Radiative torques appear to not only solve this problem, but to also explain why, and not only how, large grains $(a \gtrsim 0.1 \mu \mathrm{m}$ ) align while small grains don't (as first established by Kim \& Martin, 1995).

This promising mechanism was first discovered by Dolginov \& Mitrofanov (1976). Considering a grain exhibiting a difference in optical cross-section for right-handed and lefthanded photons, they noticed that scattering of unpolarized light by the grain resulted in its spin-up, which leads to its alignment with the surrounding magnetic field. However, they did not have the necessary tools to quantify this effect and, as a result, their pioneering work was mostly ignored in the following 20 years. Draine \& Weingartner (1996) realized that this mechanism could be studied with a modified version of the DDSCAT code by Draine \& Flatau (1994). In particular, this work, along with follow-up studies by Draine \& Weingartner (1997) and Weingartner \& Draine (2003), demonstrated that the magnitude of radiative torques is very substantial for the grain shapes they study. It is worth pointing out that the spin-up of grains by radiative torques was later observed in laboratory conditions by Abbas et al. (2004). 
However, radiative torques have the inconvenience of depending on many parameters, such as grain shape, grain size, radiation wavelength, grain composition, and the angle between the radiation direction and the local magnetic field. It is therefore difficult to compute their effect in general, and empirical studies have so far been focused on demonstrating the radiative torque effect for specific values of these parameters. As a result, it has been claimed that the predictive power of this theory was limited, which is true if a "brute force" approach is used to explore the relevant parameter space. An alternative is to use analytical models, such as the one proposed by Lazarian \& Hoang (2007), to describe the effects of radiative torques.

\subsection{Analytical Model of Radiative Torques}

Lazarian \& Hoang (2007) demonstrated that the simple model shown in Fig. 4 can be used to accurately calculate the radiative torques exerted by an incoming radiation on a dust grain. In this model, grains are assumed to be ellipsoidal, and each grain is equipped with a mirror attached to its side. A grain can be both "left-handed" and "right-handed" (from the point of view of the incoming radiation) depending on the orientation of its mirror with respect to the incident light - the grain shown in Fig. 4 is left-handed, and would become right-handed if the mirror were rotated by $90^{\circ}$ in $\alpha$. Radiative torques are then computed under the assumption that geometric optics apply, and compared to numerical calculations performed by DDSCAT.

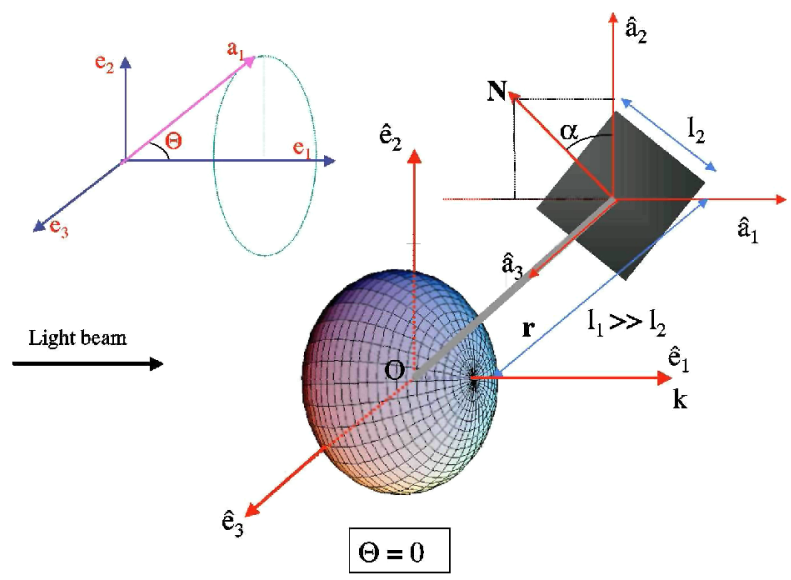

Figure 4: Lazarian \& Hoang (2007) model of a "helical" grain consisting of a spheroidal grain with an inclined mirror attached to it. This model can be used to simplify the calculation of radiative torques (see text). $\mathbf{k}$ indicates the direction of propagation of the incoming radiation, and $\Theta$ the angle between the incident light and the polar axis of the ellipsoidal grain.

The comparison is done for light propagating in the direction of the grain's short axis $\hat{e}_{1}$, while the grain's angular momentum $\mathbf{J}$ is in the $\left(\hat{\mathbf{e}}_{1}, \hat{\mathbf{e}}_{2}\right)$ plane. For the purpose of modeling grain alignment, only torques in that plane then matter - a torque in the $\hat{\mathbf{e}}_{3}$ direction would 
induce grain precession negligible compared to the Larmor precession of the grain in the interstellar magnetic field (see Tab. 1 of Lazarian (2007) for the time scales involved). As a result, the model only needs to accurately reproduce the components $Q_{\mathrm{e} 1}$ and $Q_{\mathrm{e} 2}$ of the torque $\mathbf{Q}$ along $\hat{\mathbf{e}}_{1}$ and $\hat{\mathbf{e}}_{2}$, which it does. Defining $\Theta$ as the angle between the magnetic field and the radiation direction, Lazarian \& Hoang (2007) indeed showed that the deviation of the torques calculated numerically for irregular grains by DDSCAT from their analytical predictions is always modest at the wavelengths of interest. As an illustration, Fig. 5 shows

$$
\left\langle\Delta^{2}\right\rangle\left(Q_{\mathrm{e} 2}\right) \equiv \frac{1}{\pi\left(Q_{\mathrm{e} 2}^{\max }\right)^{2}} \int_{0}^{\pi}\left[Q_{\mathrm{e} 2}^{\mathrm{DDSCAT}}(\Theta)-Q_{e 2}^{\operatorname{model}}(\Theta)\right]^{2} \mathrm{~d} \Theta,
$$

which characterizes the deviation in the $\hat{\mathbf{e}}_{2}$ direction, as a function of wavelength for various grain shapes and sizes. The value of $\Delta^{2}$ remains small no matter the values of these parameters, which indicates a remarkable agreement between analytical model and numerical calculations.

In fact, Lazarian \& Hoang (2007) showed that, for a given grain, radiative torque alignment is fully determined by the ratio $q \equiv Q_{\mathrm{e} 1}^{\max } / Q_{\mathrm{e} 2}^{\max }=Q_{\mathrm{e} 1}(0) / Q_{\mathrm{e} 2}(\pi / 4)$. This enormously simplifies the calculations of radiative torques since one does not need to calculate two functions, $Q_{\mathrm{e} 1}(\Theta)$ and $Q_{\mathrm{e} 2}(\Theta)$, but only two numbers, $Q_{\mathrm{e} 1}(0)$ and $Q_{\mathrm{e} 2}(\pi / 4)$, to characterize the alignment. $q$ is therefore as important to grain alignment as grain axial ratios are to determining the polarized radiation by aligned interstellar dust grains.



Figure 5: Numerical comparison of the radiative torques calculated with DDSCAT for irregular grains with the results provided by the analytical model ("AMO") described in Sec. 6.1 using the wavelength-dependent $\chi^{2}$ estimator defined by Eq. (5). $a_{\text {eff }}$ refers to the radius of a sphere with the same volume as the ellipsoid used in the analytical model (the index is omitted in the text), and the considered shapes are DDSCAT shapes (see Lazarian \& Hoang, 2007).

\subsection{Alignment Configuration}

Observations indicate that interstellar grains tend to align with long axes perpendicular to the local magnetic field, a fact once frequently used to argue that the Davis-Greenstein mechanism had to be responsible for the alignment. Radiative torques also explain this configuration. 
Interstellar grains indeed experience internal relaxation that tends to make them rotate about their axis of maximal moment of inertia, which, in the case of an ellipsoidal grain, is the grain's short axis. Thus, the grain's angular momentum $\mathbf{J}$ is perpendicular to its long axis, and it is therefore sufficient to follow its dynamics to determine the alignment configuration. Let us call the component of the torque parallel (respectively, perpendicular) to $\mathbf{J}$ the spin-up torque $\mathbf{H}$ (respectively, the alignment torque $\mathbf{F}$ ). The angular momentum $\mathbf{J}$ is precessing about the magnetic field $\mathbf{B}$ due to the magnetic moment of the grain (Dolginov \& Mitrofanov, 1976). It is possible to show that, when the angle $\xi$ between $\mathbf{J}$ and $\mathbf{B}$ tends to zero (or, by symmetry, $\pi$ ), the alignment torque $\mathbf{F}$ averaged over one Larmor precession of $\mathbf{J}$ about $\mathbf{B}$ also tends to zero (see Lazarian \& Hoang, 2007). In other words, $\langle\mathbf{F}\rangle_{\xi}$ vanish as $\xi \rightarrow 0$ or $\pi$ irrespectively of the radiative torques' functional form, and the points $\xi=0$ and $\pi$ are attractors.

This qualitative argument is quite general, but it does not address the question of whether there are other stationary points, and in particular of whether the alignment can also happen with $\mathbf{J}$ perpendicular to $\mathbf{B}$. To answer this question, one has to consider the behavior of the functions $Q_{\mathrm{e} 1}(\Theta)$ and $Q_{\mathrm{e} 2}(\Theta)$. The analysis in Lazarian \& Hoang (2007) shows that there is, indeed, a range of angles between the direction of radiation and the magnetic field for which grains tend to align in the "wrong" way, i.e., with long axes parallel to the magnetic field. However, this range is very narrow around $\pi / 2$, and therefore not typical of conditions in the interstellar medium. Moreover, such an alignment corresponds to positions for which the spin-up torque $\mathbf{H}$ is negative, which induces grain alignment with low angular momentum. Grain thermal wobbling at low- $J$ attractor points leads to variations in $\xi$ that typically exceed the range of angles in which "wrong alignment" happens (Lazarian, 1994; Lazarian \& Roberge, 1997). Therefore, grains always align with long axes perpendicular to the magnetic field.

\subsection{Testing Quantitative Predictions with CMBPol}

In Sec. 6.1, we pointed out that, when induced by radiative torques, grain alignment is fully characterized by the value of the parameter $q$. For typical interstellar conditions, this process dominates over all other alignment-inducing torques, and alignment of interstellar dust grains should therefore be governed by $q$ independently of the magnitude of radiative torques.

Fig. 6 shows how a given value of $q$ can be translated into information about grain alignment. In particular, depending on the angle $\psi$ between the incident radiation and the local magnetic field, grains align with $\xi$ taking the value of high- $J$ or low- $J$ attractor points. In the former case, alignment with the local magnetic field is perfect. However, when $\xi$ takes the value of a low- $J$ attractor point, the alignment is unstable, which leads to a degree of alignment of only $\sim 20 \%$ to $50 \%$. This situation is actually the most common for relevant values of $q$, meaning that most grains align subthermally. This conclusion is quite different from the assumption by Draine \& Weingartner (1996) that in the presence of radiative torques most interstellar grains should rotate with $T_{\text {rot }} \gg T_{\text {gas }}$, and therefore align suprathermally with $\mathbf{B}$.

When superparamagnetic grains, i.e., grains with enhanced paramagnetic relaxation, 


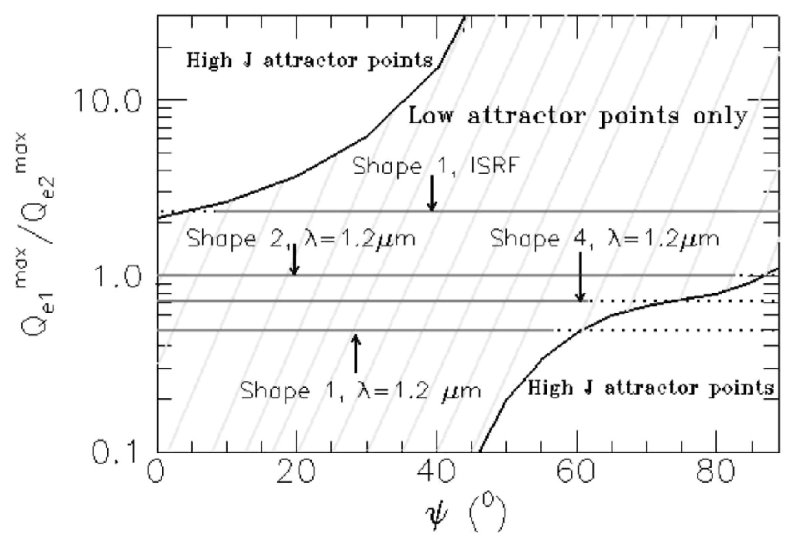

Figure 6: Type of available attractor points in the $(q, \psi)$ parameter space (see text for the definition of these parameters). When both high- $J$ and low- $J$ attractor points are present, grains eventually perfectly align in the state of highest $J$. If only low- $J$ attractor points are present, the alignment is partial. "ISRF" refers to the local interstellar radiation field, and the various shapes considered here are DDSCAT shapes (see Lazarian \& Hoang, $2007)$.

are considered, the conclusion is quite different. These grains, invoked by Jones \& Spitzer (1967) in the context of paramagnetic alignment (see also arguments in support of their existence in Bradley, 1994; Martin, 1995; Goodman \& Whittet, 1995), indeed exhibit low- $J$ attractor points when subjected to the diffuse interstellar radiation field, as for ordinary paramagnetic grains, but a more stable high- $J$ attractor point also always exists. Therefore, if a grain aligns at a low- $J$ attractor point, gaseous collisions ensure that its state will change to that of the high- $J$ attractor point. Thus, rather unexpectedly, intensive paramagnetic relaxation changes the rotational state of dust grains, enabling them to rotate more rapidly, and leading to perfect suprathermal alignment with the local interstellar magnetic field.

Although we mentioned that radiative torques dominate over all other alignment-inducing torques in the diffuse interstellar medium, this might not be the case for paramagnetic grains undergoing $\mathrm{H}_{2}$ formation at their surface. The resulting "pinwheel" torques (Purcell, 1979) can indeed create new high- $J$ attractor points (Hoang \& Lazarian, 2008). As a result, provided that $\mathrm{H}_{2}$ torques are as strong as considered in Purcell (1979) and subsequent papers by Spitzer \& McGlynn (1979), Lazarian (1995), and Lazarian \& Draine (1997), higher degrees of polarization are expected in regions of higher atomic hydrogen content. Detection of these variations would be highly interesting since it would rule out the superparamagnetic dust grains hypothesis for which the alignment should be perfect irrespectively of other factors.

Thanks to the existence of these quantitative predictions, the theory of grain alignment is now becoming testable. Lazarian (2007) reviews some of the first tests the theory of radiative torque alignment has already passed. Since then, it has also been tested in dense environments such as molecular clouds (Andersson \& Potter, 2007; Whittet et al., 2008). The current agreement between theory and observations is encouraging, and the mere fact 
that quantitative predictions exist that can be tested shows the tremendous progress made in the study of grain alignment over the course of the last few years. However, additional tests are required, and CMBPol offers a great opportunity to further constrain theories of grain alignment. By improving our understanding of the structure of the Galactic magnetic field (Sec. 4.2), and our ability to measure polarized dust emission (Sec. 5.5) and morphologically trace foregrounds (which we discuss in Sec. 8.3), CMBPol will indeed make it possible to test radiative torque alignment predictions at multiple frequencies and in various environments.

In the meantime, observations at smaller scales, and later on with the Planck satellite, will improve our knowledge of grain alignment. With a better understanding of the degree of alignment of various types of grain, it will become possible to make more robust predictions of the expected polarized dust emission at CMB frequencies by combining fits to data at other wavelengths with theory, therefore improving on the Draine \& Fraisse (2008) results. These improved templates will be helpful in generating more accurate sky models used as input to study how to best separate foreground emissions from cosmological signal (for more details on this topic, we refer the reader to the companion paper by Dunkley et al., 2008a).

\section{Heliospheric Dust Emission}

\subsection{Basic Properties from In Situ Observations}

Circumheliospheric interstellar material (CHISM) flows through the heliosphere with a heliocentric velocity of $26.3 \mathrm{~km} / \mathrm{s}$ from the upwind direction $\ell \sim 3.5^{\circ}, b \sim 15.2^{\circ}$. Approximately $0.3 \%$ to $0.9 \%$ of the CHISM mass is contained in interstellar dust grains with masses ranging from $10^{-15}$ to $10^{-10} \mathrm{~g}$ (Grün et al., 1994; Frisch et al., 1999; Landgraf et al., 2000; Witte, 2004; Slavin \& Frisch, 2008), corresponding to radii $a$ between $\sim 0.04 \mu \mathrm{m}$ and $2 \mu \mathrm{m}$ for spherical grains with density $2.5 \mathrm{~g} / \mathrm{cm}^{3}$. The dynamic interaction of an interstellar dust grain (ISDG) with the heliosphere depends on the ratio of radiation pressure to gravitational force $(\beta)$, as well as on the Lorentz force, which is a function of grain velocity, charge-to-mass ratio $(Q / m)$, and solar wind magnetic field. Consequently, the distribution of ISDGs in the heliosphere varies between upwind and downwind, i.e., between Northern and Southern ecliptic regions, and with the 22-year solar magnetic activity cycle. Ulysses observed a varying flux of grains through the inner heliosphere of $0.6-2 \times 10^{-8}$

$\mathrm{cm}^{-2} \mathrm{~s}^{-1}$ (Landgraf et al., 2003). The composition and optical properties of ISDGs interacting with the heliosphere are consistent with astronomical silicates such as olivine; organic particles may be excluded by the overabundance of carbon in CHISM gas (Landgraf et al., 1999; Slavin \& Frisch, 2008). However, Helios data inside 1 AU showed large ISDGs (radii between 1 and $2 \mu \mathrm{m}$ ), with mixed composition grains containing both silicates and carbonaceous material (Altobelli et al., 2006).

The dependence of grain propagation on $Q / m$ and $\beta$ leads to several characteristics of the spatial distribution of ISDGs in the heliosphere (Kimura \& Mann, 1998; Frisch et al., 1999; Landgraf et al., 2003; Frisch, 2007; Krüger \& Grün, 2008): (a) There is a severe reduction in the number of grains with radii $a \lesssim 0.2 \mu \mathrm{m}$ penetrating to within $5 \mathrm{AU}$ of the Sun when compared to the initial interstellar power law distribution (see, e.g., the simulations shown 

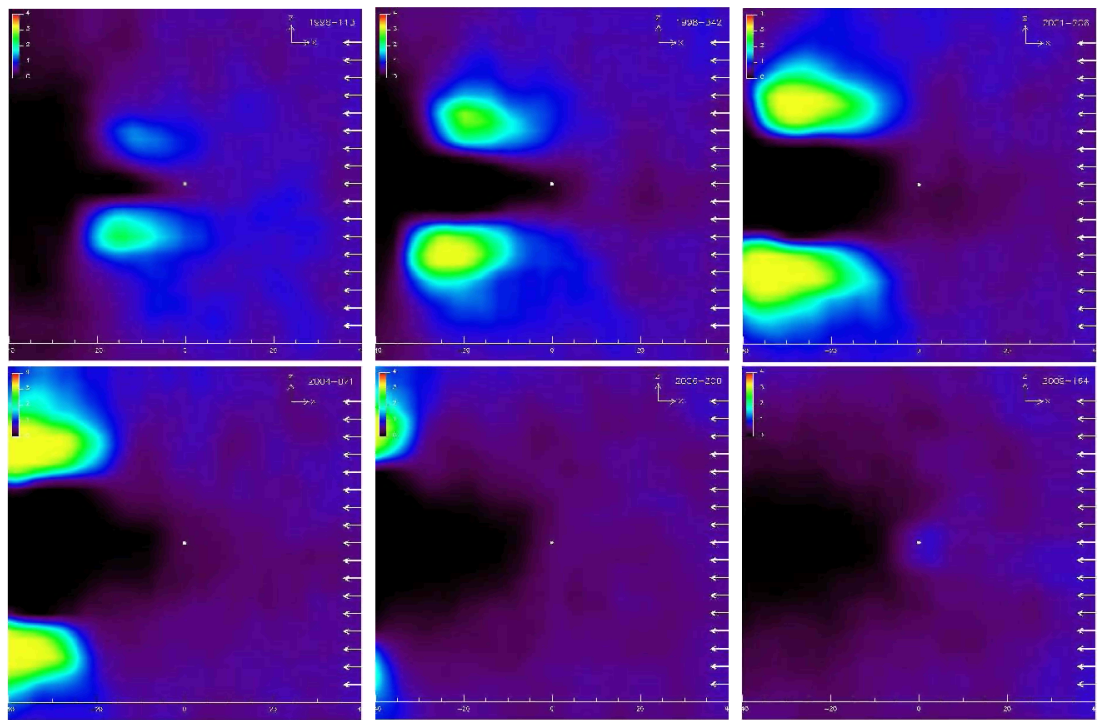

Figure 7: Simulations by Landgraf (2000) of the distribution of interstellar dust grains with radii $a \sim 0.2 \mu \mathrm{m}$ in the $\mathrm{X}-\mathrm{Z}$ plane (see below) are shown for the years 1996, 1998.5, 2001, 2003.5, 2006, 2008.5 (upper left through lower right). The 1996 figure (upper left) shows the grain distribution during solar minimum defocusing conditions, while the 2006 figure (lower middle) shows the distribution during solar minimum focusing conditions. ISDGs move through the heliosphere at velocities of about $5 \mathrm{AU} / \mathrm{yr}$, so the dust distribution in each panel reflects the influence of the solar cycle over several years prior to that date. The 1996 solar cycle phase is similar to that expected for a $2018 \mathrm{CMBPol}$ launch date. Individual plots are $80 \mathrm{AU} \times 80 \mathrm{AU}$, with the Sun at the center. The $\mathrm{X}$-axis is directed towards the upwind direction of the CHISM flowing through the heliosphere, while the $\mathrm{Z}$-axis is perpendicular to the $\mathrm{X}$-axis and nominally shows the solar rotation axis since the upwind direction is $5^{\circ}$ from the ecliptic plane. The color scale is given in units of the dust density in the diffuse interstellar medium, and ranges from 0 (deep purple) to 4 (red).

in Fig. 7). (b) Large grains, $a \gtrsim 0.5 \mu \mathrm{m}$, on hyperbolic orbits are gravitationally focused into a tail downwind of the Sun (Grogan et al., 1996; Landgraf, 2000). (c) A gap in the distribution of ISDGs with mass between $10^{-14}$ and $10^{-13} \mathrm{~g}$ is found $\sim 2-4$ AU from the Sun due to the effect of radiation pressure (Landgraf et al., 1999). (d) Small grains with masses $m \lesssim 10^{-13} \mathrm{~g}$ are alternately focused towards, or away from, the ecliptic plane depending on solar magnetic polarity (Grogan et al., 1996; Landgraf, 2000). (e) There is a time lag of $\sim 4-6$ years between solar cycle phase and its influence on inner heliosphere ISDGs because of the nominal $5 \mathrm{AU} / \mathrm{yr}$ grain propagation velocity through the heliosphere. (f) The CHISM contains large ISDGs, $m \gtrsim 10^{-12} \mathrm{~g}$, that are difficult to explain in the context of the extinction properties of more heavily obscured sightlines (Frisch et al., 1999; Landgraf et al., 2000; Draine, 2008). Only properties (b), (e), and (f) above are relatively invariant with the solar cycle phase.

Several current or proposed spacecraft missions have the potential to return data on ISDGs in the heliosphere by the time CMBPol flies. The only spacecraft presently capable 
of making in situ measurements of ISDGs beyond $1 \mathrm{AU}$ from the Sun is Cassini. In situ data collected during the same time period by Ulysses and Cassini find consistent results, detecting grains with radii $\sim 0.4 \mu \mathrm{m}$ on hyperbolic orbits with fluxes $\lesssim 2 \times 10^{-9} \mathrm{~cm}^{-2} \mathrm{~s}^{-1}$ (see the analysis by Altobelli et al., 2007). The Cassini mission has now been extended to 2010; the further extension of this mission to $\sim 2016$ (depending on spacecraft consumables) would provide in situ data on ISDGs for a similar solar magnetic polarity as expected for the CMBPol launch time. Proposed interstellar dust missions include DUst Near Earth (DUNE) (Grun \& Srama, 2006) and SAmple Return of Interstellar Matter (SARIM) (Srama et al., 2008). DUNE will have the capability of acquiring the mass distribution, charge state, elemental and isotopic composition, and velocity vectors of ISDGs near 1 AU, while SARIM will return interstellar and interplanetary dust grains for laboratory analysis, providing "ground-truth" on ISDGs.

\subsection{Emission Characteristics and CMBPol Observations}

The heating of interstellar dust grains $1-90 \mathrm{AU}$ from the Sun is dominated by solar radiation, yielding a grain temperature in $\mathrm{K}$ at distance $R$ from the Sun of $\sim 278 R^{-0.5}$, where $R$ is in units of AU. The emission of these grains is expected to be $\sim 10^{-3}$ of zodiacal light emission (Mukai, 1981; Frisch et al., 1999). For some inner heliosphere viewing configurations and solar cycle phases, strong asymmetries in ISDG emissions are expected for leading versus trailing sightlines with respect to the Earth's orbital motion, and high versus low ecliptic latitude lines-of-sight. Grogan et al. (1996) predict emissions of $\sim 0.1$ $\mathrm{MJy} / \mathrm{sr}$ at $12 \mu \mathrm{m}$ for sightlines that view the hotter ISDGs near the Sun during a focusing phase. Smaller grains at low solar elongation angles dominate this emission. Fixsen \& Dwek (2002) find the ratio of the $12 \mu \mathrm{m}$ to $340 \mu \mathrm{m}$ flux to be $I_{12} / I_{340} \sim 500$ for zodiacal light. Full models of the emissions from heliospheric interstellar dust are under development (Brahman et al., in preparation), but, based on this value of $I_{12} / I_{340}$, we expect that the emission from interstellar dust grains in the heliosphere could be as bright as $0.2 \mathrm{kJy} / \mathrm{sr}$ during a focusing cycle.

The distribution of ISDGs inside of the heliosphere is highly asymmetric around the Sun. Figure 7 shows simulations of the distribution of $a=0.2 \mu \mathrm{m}$ ISDGs in the X-Z plane (see caption for definition) at $\sim 2.5$ year intervals starting in 1996 (Landgraf, 2000). During the year 2018, which could see the launch of the CMBPol satellite, conditions similar to those shown for the year 1996 are expected. For comparison, the COBE data were collected at the end of the 1980's focusing cycle, while WMAP, launched in 2001, has witnessed the phases shown in the last four panels of Fig 7 (starting by the top right panel). The flux from $0.2 \mu \mathrm{m}$ ISDGs in the inner heliosphere varies by over an order of magnitude between solar minimum and solar maximum conditions (Landgraf et al., 2003). As a result, a CMBPol mission is likely to detect time variability in the emission from heliospheric dust if it is operated for a long enough time. This result would be interesting by itself, since it would probe both the magnetic conditions in the heliosphere and the properties of ISDGs, but it would also be useful to the CMB analysis by signaling a possible contamination by heliospheric dust emission. 


\section{Anomalous Emissions}

\subsection{Anomalous Dust Emission}

Prior to the launch of WMAP, detailed intensity maps of the Galaxy were available at $408 \mathrm{MHz}$ (Haslam et al., 1982) and $100 \mu \mathrm{m}$ (Schlegel et al., 1998). The morphologies of these maps were notably different, reflecting the different emission mechanisms that are dominant at those frequencies: at $408 \mathrm{MHz}$, the diffuse Galactic emission is dominated by synchrotron, with large characteristic morphological features such as Loop I (the North Polar Spur); at $100 \mu \mathrm{m}$, the diffuse emission is generated primarily by dust grains emitting blackbody radiation as they are heated by the ambient interstellar radiation field. When these two maps were extrapolated to $W M A P$ frequencies, assuming reasonable power law models, it was thought that the $94 \mathrm{GHz}$ map would look morphologically very similar to the $100 \mu \mathrm{m}$ map since thermal emission from interstellar dust dominates the diffuse Galactic signal at these frequencies, while the $23 \mathrm{GHz}$ map (away from bright HII regions and filaments where free-free emission dominates) would closely resemble the synchrotron dominated $408 \mathrm{MHz}$ map. However, the WMAP $23 \mathrm{GHz}$ map looks much more like the $100 \mu \mathrm{m}$ map than like the $408 \mathrm{MHz}$ map (Hinshaw et al., 2008).

The origin of this dust-correlated emission in the low frequency WMAP channels is still not completely certain, though there is significant evidence that it arises from the smallest interstellar dust grains excited into rotational modes and emitting electric dipole radiation (Draine \& Lazarian, 1998a,b). This "spinning dust" model is currently the leading hypothesis, although "magnetic dust" emission (Draine \& Lazarian, 1999) and "dust-correlated synchrotron" emission (Bennett et al., 2003) are sometimes offered as alternative explanations. Both total intensity and polarization observations can provide useful information as to which model can best explain the observed anomalous dust-correlated emission in the WMAP data.

\subsubsection{Sorting through Candidates}

Total Intensity Prior to WMAP's launch, there was already evidence against the hypothesis of dust-correlated synchrotron emission. Using the Tenerife 10 and $15 \mathrm{GHz}$ data along with the COBE/DMR maps, de Oliveira-Costa et al. (1999) showed that the dust-correlated emission does not rise with decreasing frequency as expected for synchrotron, but rather falls off below $30 \mathrm{GHz}$, a feature which is characteristic of spinning and magnetic dust emissions. Furthermore, Finkbeiner et al. (2002) identified at least one confirmed example, L1622, of a cloud in which the spectrum of emission from 5 to $10 \mathrm{GHz}$ resembles a spinning dust spectrum.

As part of the first-year WMAP data release, Bennett et al. (2003) presented maps of the spectral index of synchrotron emission derived under the assumption that all of the anomalous emission is synchrotron. These maps have large diffuse regions in which the spectral index of synchrotron is $\beta \gtrsim-2.6$, whereas, if the synchrotron emitting electrons

are accelerated through Fermi acceleration by supernova shocks, the injected spectral index should be $\beta \sim-2.5$ (see, e.g., Gieseler \& Jones, 2000), which softens to $\beta \sim-3$ when taking into account diffusion energy losses (the total energy losses from synchrotron and inverse 
Compton scattering are proportional to $E^{2}$ ). Such a discrepancy seems to invalidate their initial assumption, a conclusion reinforced by the fact that a spectral index of $\beta \sim-3$ has been found in more rigid fits to the WMAP temperature data (Dobler \& Finkbeiner, 2008a), by combining observations at $408 \mathrm{MHz}$ and $1.42 \mathrm{GHz}$ to predict the emission at frequencies above $20 \mathrm{GHz}$ (La Porta et al., 2008), and by using only low frequency WMAP polarization data (see Kogut et al., 2007, and the Polarization discussion below for more polarization related arguments).

Targeted observations of individual diffuse clouds have also proven fruitful (see, e.g., Dickinson et al., 2006), and there is evidence that when combining WMAP data with data sets at other frequencies, diffuse, inverted spectrum emission (as opposed to the monotonically decreasing spectrum expected for synchrotron) is seen (Boughn \& Pober, 2007). Additionally, the Green Bank Galactic plane survey (Finkbeiner et al., 2004) presented very strong evidence for diffuse inverted spectrum emission between 8.35 and $14.35 \mathrm{GHz}$ outside HII regions.

Finally, Dobler \& Finkbeiner (2008b) and Dobler et al. (2008) showed that a spinning dust spectrum is completely recoverable within the WMAP frequency range. However, this

WIM ("warm ionized medium") spinning dust emission correlates more strongly with maps of $\mathrm{H} \alpha$ emission (Finkbeiner, 2003) than with maps of $100 \mu \mathrm{m}$ emission. Since $\mathrm{H} \alpha$ maps represent an emission measure, and since spinning dust emission is also a density squared process (Dobler et al., 2008), H $\alpha$ should trace spinning dust emission as well. Magnetic dust emission, on the other hand, should be proportional to density and would not be traced by $\mathrm{H} \alpha$.

Polarization Perhaps the strongest evidence against the dust-correlated synchrotron hypothesis is derived from the WMAP polarization maps presented by Page et al. (2007) and Kogut et al. (2007). Synchrotron emission is thought to be highly polarized, typically $40-70 \%$ in the presence of reasonably ordered magnetic fields, although the polarization signal can effectively be eliminated for lines-of-sight going through regions with turbulent magnetic fields (see Sec. 4.2 and, e.g., Miville-Deschenes et al., 2008). The polarized fraction of spinning dust emission, on the other hand, is expected to be smaller than $3 \%$ at the lowest $W M A P$ frequencies (Lazarian \& Draine, 2000). However, the WMAP maps of the polarized emission at $23 \mathrm{GHz}$ look morphologically very different from the corresponding total intensity map. In particular, the polarization map more closely resembles (but is not perfectly traced by) the $408 \mathrm{MHz}$ map and does not correlate very strongly with the $100 \mu \mathrm{m}$ dust map. The implication is that there is a highly polarized component that morphologically looks very much like synchrotron and a low polarization component that looks very much like dust. The polarization properties of magnetic dust are not well known (see Draine \& Lazarian, 1999).

\subsubsection{Towards a Spectral Identification}

Presently, the study of anomalous dust emission is mostly aimed at targeted searches of promising regions (e.g., dust clouds and HII regions) that may exhibit evidence for anomalous emission spectrum. These studies are being undertaken in both intensity (Dickinson 
et al., 2006) and polarization (Mason et al., 2008). The polarization studies in particular will prove very useful in determining just how much of an issue certain emission mechanisms will present when attempting to clean polarization maps to get a cosmological signal. However, we stress that determining the polarization properties of spinning dust does not exclude the possibility that other emission mechanisms (such as magnetic dust) might exist with very significant polarization fractions exhibiting a strong wavelength dependence (Draine \& Lazarian, 1999).

With the $\mathrm{H} \alpha$-correlated spinning dust signal detected in the WMAP data (see Sec. 8.1.1), progress has been made towards measuring specific parameters of the dust grains and environment (Dobler et al., 2008), but is limited by the relatively narrow frequency coverage of WMAP. Near-term and future missions will address this issue. For example, the CBand All-Sky Survey (Pearson, 2007) will map the sky in temperature and polarization at $5 \mathrm{GHz}$. This survey should determine if the dust-correlated emission continues to rise from $23 \mathrm{GHz}$ down to $5 \mathrm{GHz}$ (indicating synchrotron) or if it has fallen (indicating spinning or magnetic dust).

The Planck satellite will now launch shortly and with it will come high resolution maps at 545 and $857 \mathrm{GHz}$. At these frequencies, the foreground emission is totally dominated by thermal dust emission and not only will this give insight into the polarization properties of dust, but it will also significantly facilitate foreground cleaning, especially if the polarized fraction of dust emission is independent of wavelength (see Sec. 5). Obtaining cleaner maps is essential to deriving an unbiased spectrum of foreground emission at anomalous emission frequencies, i.e., in the range 20 - $100 \mathrm{GHz}$ (Hinshaw et al., 2007; Dobler \& Finkbeiner, 2008a).

Additionally, if the Absolute Spectrum Polarimeter (ASP, PI: A. Kogut) is funded, it will provide excellent frequency coverage from $30 \mathrm{GHz}$ to $5 \mathrm{THz}$, with over 300 spectral bins dedicated to characterizing foregrounds. Many spectral channels are highly desirable since the primary goal in foreground science is to characterize the spectrum of each emission component. Furthermore, the more well known the foreground spectra, the easier it will be to clean maps of the foreground emission to measure the cosmological signal. The shortcoming of ASP is that the angular resolution is a $2^{\circ}$ tophat, a resolution considerably lower than even WMAP's.

\subsection{The WMAP "Haze"}

In an analysis of the WMAP temperature maps, Finkbeiner (2004) detected an excess of diffuse microwaves coming from an extended region around the Galactic center. This emission has also been detected in the three-year temperature data by Dobler \& Finkbeiner (2008a) and Bottino et al. (2008). When CMB polarization data is not taken into account, this excess emission, coined "the haze," exhibits a spectrum too soft to be free-free emission, but too hard to be synchrotron from supernova shock accelerated electrons when diffusion losses are accounted for (Dobler \& Finkbeiner, 2008a). It was therefore proposed that the haze could be synchrotron emission from a population of hard electrons surrounding the Galactic center. However, Gold et al. (2008) do not find any evidence of this hardening in WMAP's observations of the polarized Galactic synchrotron emission (see, e.g., their 
Fig. 16). Although the WMAP polarization data is not yet sufficient to convincingly rule out the synchrotron nature of the haze, this discrepancy is worth investigating.

As with all inferences of foreground spectra from the WMAP data, there is substantial systematic uncertainty in the haze spectrum due to the CMB cross-correlation bias. Any CMB estimator is indeed contaminated by some residual foregrounds that then get subtracted off of each channel prior to the foreground analysis. As a result, the inferred foreground spectra are in turn contaminated by a CMB spectrum. The amplitude of the bias can be hard to estimate given that it is related to the chance morphological correlation of the foregrounds with the CMB, and, consequently, the haze spectrum is relatively uncertain. Measurements at more frequencies will therefore prove crucial to determining the fundamental nature of the haze.

Thus, the upcoming experiments discussed in the context of spinning dust emission can also play a role in characterizing the haze. In particular, with its improved foreground removal abilities, Planck will lead to a significant decrease in the amplitude of the CMB cross-correlation bias. Moreover, the amplitude of the haze in the C-BASS $5 \mathrm{GHz}$ maps will distinguish between spinning dust and synchrotron type spectrum for this excess emission. If these two surveys confirm that the haze is compatible with hard synchrotron emission, the Fermi satellite should see $\gamma$-rays from inverse Compton scattered starlight (Dobler, in preparation), which would confirm the synchrotron nature of the haze. Lastly, the many frequency channels of ASP may allow us to trace out the synchrotron spectrum (and thus the energy spectrum of the electrons) as a function of position on the sky with a resolution of a couple of degrees.

\subsection{CMBPol as a Morphological Tracer}

The CMBPol satellite has the potential to combine the most promising aspects of upcoming experiments and shed new light on both anomalous dust-correlated emission and the haze. Although the final design may only have a limited number of frequency bands, the large-scale spectral information provided by a survey like the ASP would indeed enable the separation of foreground emission and cosmological signal with high accuracy, and in particular, with insignificant CMB cross-correlation bias. Thanks to the expected very high spatial resolution of $\mathrm{CMBPol}$, one could then trace out the spectrum of individual components morphologically, which not only could provide clues regarding the origin of the haze, but is essential to constraining the parameters of an environment-dependent emission such as spinning dust.

\section{Foregrounds and CMB Lensing}

Foregrounds are a potential source of systematic error for lensing of the CMB. Quadratic lensing reconstruction with $\mathrm{CMB}$ polarization indeed generally involves taking products of 
the $E$ and $B$-mode polarization signals to compute the integral (Hu \& Okamoto, 2002)

$$
\hat{\phi}(\mathbf{l}) \equiv \int w\left(\mathbf{l}^{\prime}, \mathbf{l}-\mathbf{l}^{\prime}\right) E\left(\mathbf{l}^{\prime}\right) B\left(\mathbf{l}-\mathbf{l}^{\prime}\right) \mathrm{d}^{2} \mathbf{l}^{\prime},
$$

where $\hat{\phi}(\mathbf{l})$ is the estimator for the lensing potential $\phi(\mathbf{l}), w$ a weight function, and $\mathbf{l}$ the wavevector. There are other reconstruction techniques that give smaller error bars (see, e.g., Hirata \& Seljak, 2003), but, for the purpose of this discussion, we focus on the quadratic methods as they are easier to analyze. In general, three types of measurements concern us:

- the galaxy-lensing potential cross-power spectrum,

- the lensing potential auto-power spectrum, and

- the low-l $B$-mode power spectrum after correcting for lensing.

The last of these is the most directly important for the community's quest to detect a primordial gravitational wave signal, while the first two are relevant for low-redshift (e.g., dark energy and neutrino mass) studies. The companion paper by Smith et al. (2008) focuses on the lensing auto-power spectrum. We assess the foreground situation for this particular case.

As Eq. (6) involves a product of $E$ and $B$-mode polarizations, the estimator's power spectrum is a trispectrum containing two factors of the $E$-mode polarization and two of the $B$, with both "disconnected" terms (involving the product of two power spectra) and "connected" (i.e., non-Gaussian) terms. The disconnected terms depend on the product of the CMB $C_{l}^{E E}$ and the foreground power spectrum $C_{l}^{B B}$. The latter are much harder to estimate as they depend on the polarized foreground trispectrum, which is mostly unknown at the present time.

Since lensing reconstruction uses mainly the small-scale polarization, our expectation is that extragalactic foregrounds (point sources) will dominate. In this sense we are fortunate, in that for point sources with random polarization angles it is possible to write the relevant trispectrum in terms of three quantities: (i) the polarized flux-squared-weighted integral of the unmasked source counts, $I_{2}$; (ii) the fourth-power-weighted integral, $I_{4}$; and (iii) the angular clustering power spectrum of the point sources, $C_{l}^{\mathrm{s}}$. While the polarized source counts at relevant frequencies are presently unknown, we can estimate reasonable ranges for these parameters. More importantly, the dependence on a single function raises the hope that determination of the foreground parameters may be possible by examining the full configuration dependence of the trispectrum, a possibility yet to be investigated. This rather optimistic take on the foreground trispectrum should however be tempered by some "known unknowns."

- Point sources have a different spectrum than the CMB, which means that in a broadband CMB map they are not truly "pointlike."

- Extragalactic microwave sources may not have random polarization angles. For example, tidal torques may align disk galaxies with each other, resulting in a net alignment of magnetic fields. The current data are insufficient to constrain such a contamination. 
- Even in "clean" parts of the sky, there is Galactic emission which may contain nonGaussian small-scale polarized structure with a complicated configuration dependence.

These problems will have to be continuously re-assessed as we continue with small-scale CMB polarization experiments. For example, Planck will measure the small angular scale Galactic dust emission and empirically constrain its $E / B$ structure and configuration dependence, which cannot be accurately predicted from theory or measured with WMAP. We also expect that prior to CMBPol, a number of ground-based experiments will make the first characterization of the lensing $B$-mode peak, and provide information on the polarization fractions and polarized SEDs of point sources at relevant frequencies. Because of the frequency dependence of the beams, this information will be crucial even if the use of angular information (configuration dependence) turns out to be a viable approach to lensing foreground removal.

\section{Summary and Conclusions}

The main conclusions of this paper are as follows:

1. Our current knowledge of the large-scale structure of the Galactic magnetic field comes mostly from radio observations of the Galactic synchrotron emission (Sec. 2.1.1) and Faraday rotation (Sec. 2.1.2), with the former providing information about the structure of the field in the plane of the sky, while the latter encodes information about the line-of-sight component of the field. Other techniques (Sec. 2.1.4), such as observations of polarized dust emission, starlight polarization, and Zeeman splitting, complete this large-scale picture by probing the field on smaller scales, e.g., in molecular clouds, massive star forming regions, and low-dust extinction environments. Despite this combination of techniques, the large-scale structure of the field is still poorly constrained (Sec. 2.2.1), with, in particular, no real agreement as to the number and location of large-scale reversals (Fig. 1). In addition, very little is currently known about both the small-scale field (Sec. 2.2.2) and the halo field (Sec. 2.2.3).

2. Combining multiple probes of the Galactic magnetic field, such as observations of total and polarized synchrotron emissions, free-free emission, and Faraday rotation, to simultaneously fit for various parameters describing the magnetized interstellar medium can lift degeneracies between them (Sec. 3), thereby improving constraints on their values.

3. Knowing the structure and strength of the Galactic magnetic field is a pre-requisite to understanding both the total and polarized synchrotron emissions (Sec. 2.1.1) and the polarized dust emission (Sec. 5.6). The progress we will make towards characterizing the field before CMBPol flies will therefore be of tremendous help in separating Galactic foregrounds from cosmological signals. Combining RM synthesis (Sec. 2.1.3) and radio polarimetric surveys with wide frequency and spatial coverage will greatly improve our knowledge of the field by mapping it in 3 dimensions and enabling the use of RM grids (Sec. 4.1). In particular, detailed studies of the number and locations 
of large-scale field reversals, the possible inclination of the field with respect to the Galactic plane, the vertical structure of the field, and the properties of the turbulent field on a wide range of scales will be carried out by the end of the next decade. Data from the Auger experiment may additionally allow probing the large-scale structure of the Galactic magnetic field without having to rely on a model for the electron density in our galaxy (Sec. 2.1.4).

4. CMBPol will shed new light on the structure of the Galactic magnetic field through higher resolution and sensitivity observations of the total and polarized synchrotron emissions from the Galaxy than WMAP and Planck (Sec. 4.2). These observations are unaffected by Faraday rotation and therefore complement RM data by probing the sky-projected component of field. However, the sensitivities of Planck and WMAP will not be high enough for them to accurately determine the characteristics of the polarized synchrotron emission at intermediate and high Galactic latitudes. With 10 times higher sensitivity, CMBPol would be able to constrain the field at these latitudes, and in particular its vertical structure and its halo component. Higher resolution will also allow CMBPol to study turbulent structures in the magneto-ionized medium, and to potentially trace magnetic fields from the diffuse ISM into small-scale structures.

5. Models of interstellar dust (Sec. 5.2) based on observations of the Galactic extinction and polarization of starlight (Sec. 5.1) can be used to predict the diffuse polarized far-infrared and submillimeter dust mission from our galaxy (Sec. 5.3). However, predictions are different for models involving different species of polarization-contributing dust grains (Fig. 3). Although this might make separating polarized dust emission from cosmological signal more complicated (Sec. 5.4), it also offers the opportunity of improving our knowledge of the properties of interstellar dust grains with CMBPol observations (Sec. 5.5).

6. Starlight polarization data can provide useful Galactic magnetic field templates to trace the polarized dust emission (Sec. 5.6), but few data points are available at latitudes of interest to CMB studies, typically $|b| \gtrsim 10^{\circ}$. No currently underway or planned experiment addresses this issue, since all of them focus either on regions of the Galactic plane or on dense small-scale environments. We believe obtaining this data by the time CMBPol flies should be a high priority item.

7. Alignment of dust grains with the Galactic magnetic field is the physical process behind both polarized dust emission and starlight polarization. The radiative torque theory of grain alignment (Sec. 6) seems to be able to explain why and how dust grains in the interstellar medium align the way observations indicate (Sec. 6.2), but the most exciting feature of this theory is its ability to make testable predictions (Sec. 6.3) thanks to simple models accurately reproducing complex numerical calculations (Sec. 6.1). CMBPol's high sensitivity and resolution, combined with starlight polarization information, will therefore allow testing the theory of radiative torques by observing the diffuse polarized dust emission from the Galaxy in numerous independent patches of the sky (Sec. 5.5). 
8. The distribution of dust grains in the heliosphere, and therefore the corresponding farinfrared and submillimeter dust emission, vary cyclically due to the varying magnetic conditions originating from the solar activity cycle (Fig. 7). Although more precise estimates are needed, CMBPol could detect these fluctuations (Sec. 7.2), thereby constraining the magnetic conditions in the heliosphere and the properties of heliospheric dust.

9. Two anomalous emissions were detected in the WMAP data. Dust-correlated emission in its low frequency channels is likely to be spinning or magnetic dust emission (Sec. 8.1), whereas the origin of an excess emission of microwaves towards the Galactic center, dubbed "the haze" (Sec. 8.2), is still unknown but could be synchrotron radiation from a population of hard electrons. A definitive identification of these two emissions likely requires observing their spectra, an effort ground-based experiments, e.g., C-BASS, and dedicated satellite missions, such as the ASP, will contribute to (Sec. 8.1.2). CMBPol's high resolution will enable us to trace these spectra morphologically (Sec. 8.3). This is essential to determining the parameters of spinning dust emission, which strongly depend on environment, and it might give us clues as to the possible origin of the haze.

10. Finally, extra-Galactic sources are a potential contaminant for studies of CMB lensing (Sec. 9), but not much is currently known about the polarized source counts at CMB frequencies, or the spectra and intrinsic polarizations of these sources. The input of ground-based experiments on these questions will be essential to improve our measurements of CMB lensing (see more details in the companion paper by Smith et al., 2008).

Detecting a primordial B-mode polarization signal would have dramatic consequences for our understanding of the phenomena that took place in the early Universe and led to the necessary conditions for it to evolve into the Universe we observe today (see the companion paper by Baumann et al., 2008a). Being able to do so will not only require advanced CMB data analysis techniques (see the companion paper by Dunkley et al., 2008a), but also an in-depth understanding of how Galactic [and, for some purposes (see, e.g., the companion paper by Smith et al., 2008), extra-Galactic] foregrounds behave. Taking this synergy to the next level necessitates coordinated and collaborative efforts by both the CMB and the ISM communities, each of which, as we hope to have shown throughout this document, will highly benefit from.

\section{Acknowledgments}

This research was partly funded by NASA Mission Concept Study award NNX08AT71G S01. We also acknowledge the organizational work of the Primordial Polarization Program Definition Team (PPPDT). AAF was supported by NSF grant AST-0707932 and Princeton University. PCF acknowledges support from NASA grant NNX08AJ33G, AL from NSF grant AST-0507164 and the NSF Center for Magnetic Self-Organization in Laboratory and Astrophysical Plasmas, and AMM from FAPESP and CNPq. AAF would like 
to thank D. P. Finkbeiner and J. E. Vaillancourt for their contributions to the CMBPol Theory Workshop held at Fermilab in June 2008, L. A. Page for stimulating discussions, D. N. Spergel for comments on a draft version of this paper, and R. H. Lupton for making the SM plotting program available to him. AMM is grateful to all members of the IAG Polarimetry Group for their continued support. Typesetting of this document was made easier by the use of NASA's Astrophysics Data System Bibliographic Services and of the AASTEX package. All the Planck-related numbers and information referred to in this document are from the Planck scientific program (also known as "blue book") available online in PDF format at http://www.rssd.esa.int/planck/.

\section{References}

Abbas, M. M. et al. 2004, ApJ, 614, 781

Abraham, J. et al. 2007, Science, 318, 938

Altobelli, N., Dikarev, V., Kempf, S., Srama, R., Helfert, S., Moragas-Klostermeyer, G., Roy, M., \& Grün, E. 2007, Journal of Geophysical Research (Space Physics), 112, 7105

Altobelli, N., Grün, E., \& Landgraf, M. 2006, A\&A, 448, 243

Anderson, C. M. et al. 1996, AJ, 112, 2726

Andersson, B.-G., \& Potter, S. B. 2007, ApJ, 665, 369

Andreasyan, R. R., \& Makarov, A. N. 1988, Astrophysics, 28, 247

Arendt, R. G. et al. 1998, ApJ, 508, 74

Baumann, D. et al. 2008a, ArXiv e-prints, 0811.3919

- 2008b, ArXiv e-prints, 0811.3911

Beck, R. 2001, Space Science Reviews, 99, 243

Beck, R. 2007, in EAS Publications Series, Vol. 23, Sky Polarisation at Far-Infrared to Radio Wavelengths, ed. M.-A. Miville-Deschênes \& F. Boulanger, 19-36

Beck, R., Brandenburg, A., Moss, D., Shukurov, A., \& Sokoloff, D. 1996, ARA\&A, 34, 155

Beck, R., \& Gaensler, B. M. 2004, New Astronomy Review, 48, 1289

Beck, R., \& Krause, M. 2005, Astronomische Nachrichten, 326, 414

Beck, R., Shukurov, A., Sokoloff, D., \& Wielebinski, R. 2003, A\&A, 411, 99

Bennett, C. L. et al. 2003, ApJS, 148, 97

Benoit, A. et al. 2004, A\&A, 424, 571

Berkhuijsen, E. M., Brouw, W. N., Muller, C. A., \& Tinbergen, J. 1964, Bull. Astron. Inst. Netherlands, 17,465

Berkhuijsen, E. M., Mitra, D., \& Mueller, P. 2006, Astronomische Nachrichten, 327, 82

Bock, J. et al. 2008, ArXiv e-prints, 0805.4207

Bottino, M., Banday, A. J., \& Maino, D. 2008, MNRAS, 389, 1190

Boughn, S. P., \& Pober, J. C. 2007, ApJ, 661, 938 
Boulares, A., \& Cox, D. P. 1990, ApJ, 365, 544

Bradley, J. P. 1994, Science, 265, 925

Brentjens, M. A., \& de Bruyn, A. G. 2005, A\&A, 441, 1217

Brouw, W. N., \& Spoelstra, T. A. T. 1976, A\&AS, 26, 129

Brown, J. C., Haverkorn, M., Gaensler, B. M., Taylor, A. R., Bizunok, N. S., McClure-Griffiths, N. M., Dickey, J. M., \& Green, A. J. 2007, ApJ, 663, 258

Brown, J. C., Taylor, A. R., \& Jackel, B. J. 2003a, ApJS, 145, 213

Brown, J. C., Taylor, A. R., Wielebinski, R., \& Mueller, P. 2003b, ApJ, 592, L29

Burn, B. J. 1966, MNRAS, 133, 67

Burstein, D., \& Heiles, C. 1982, AJ, 87, 1165

Carciofi, A. C., Magalhães, A. M., Leister, N. V., Bjorkman, J. E., \& Levenhagen, R. S. 2007, ApJ, $671, \mathrm{~L} 49$

Chandrasekhar, S., \& Fermi, E. 1953, ApJ, 118, 113

Chiar, J. E. et al. 2006, ApJ, 651, 268

Clayton, G. C. et al. 1992, ApJ, 385, L53

Clemens, D. P., Pinnick, A., Pavel, M., Jameson, K., Carveth, C., \& Taylor, B. 2007, in Bulletin of the American Astronomical Society, Vol. 38, 761

Cordes, J. M., \& Lazio, T. J. W. 2002, ArXiv Astrophysics e-prints, astro-ph/0207156

- 2003, ArXiv Astrophysics e-prints, astro-ph/0301598

Crutcher, R., Heiles, C., \& Troland, T. 2003, in Lecture Notes in Physics, Vol. 614, Turbulence and Magnetic Fields in Astrophysics, ed. E. Falgarone \& T. Passot, 155

Davies, R. D. 2007, Astronomische Nachrichten, 328, 436

Davies, R. D., Slater, C. H., Shuter, W. L. H., \& Wild, P. A. T. 1960, Nature, 187, 1088

Davis, L. J., \& Greenstein, J. L. 1951, ApJ, 114, 206

de Oliveira-Costa, A., Tegmark, M., Gaensler, B. M., Jonas, J., Landecker, T. L., \& Reich, P. 2008 , MNRAS, 388,247

de Oliveira-Costa, A., Tegmark, M., Gutierrez, C. M., Jones, A. W., Davies, R. D., Lasenby, A. N., Rebolo, R., \& Watson, R. A. 1999, ApJ, 527, L9

Dickinson, C., Casassus, S., Pineda, J. L., Pearson, T. J., Readhead, A. C. S., \& Davies, R. D. 2006, ApJ, 643, L111

Dobler, G., Draine, B. T., \& Finkbeiner, D. P. 2008, ArXiv e-prints, 0811.1040

Dobler, G., \& Finkbeiner, D. P. 2008a, ApJ, 680, 1222

. 2008b, ApJ, 680, 1235

Dolginov, A. Z., \& Mitrofanov, I. G. 1976, Ap\&SS, 43, 291

Draine, B. T. 2003, ARA\&A, 41, 241

- 2008, Space Science Reviews 
Draine, B. T., \& Flatau, P. J. 1994, Journal of the Optical Society of America A, 11, 1491

Draine, B. T., \& Fraisse, A. A. 2008, ArXiv e-prints, 0809.2094

Draine, B. T., \& Lazarian, A. 1998a, ApJ, 494, L19

-.1998b, ApJ, 508, 157

—. 1999, ApJ, 512, 740

Draine, B. T., \& Weingartner, J. C. 1996, ApJ, 470, 551

- $1997, \mathrm{ApJ}, 480,633$

Dumke, M., Krause, M., Wielebinski, R., \& Klein, U. 1995, A\&A, 302, 691

Dunkley, J. et al. 2008a, ArXiv e-prints, 0811.3915

- 2008b, ArXiv e-prints, 0803.0586

Dwek, E. et al. 1997, ApJ, 475, 565

Fermi, E. 1949, Physical Review, 75, 1169

Finkbeiner, D. P. 2004, ApJ, 614, 186

Finkbeiner, D. P. 2003, ApJS, 146, 407

Finkbeiner, D. P., Langston, G. I., \& Minter, A. H. 2004, ApJ, 617, 350

Finkbeiner, D. P., Schlegel, D. J., Frank, C., \& Heiles, C. 2002, ApJ, 566, 898

Fish, V. L., Reid, M. J., Argon, A. L., \& Menten, K. M. 2003, ApJ, 596, 328

Fitzpatrick, E. L. 1999, PASP, 111, 63

Fitzpatrick, E. L. 2004, in Astrophysics of Dust, ed. A. N. Witt, G. C. Clayton, \& B. T. Draine, ASP Conference Series 309, 33

Fixsen, D. J., \& Dwek, E. 2002, ApJ, 578, 1009

Fosalba, P., Lazarian, A., Prunet, S., \& Tauber, J. A. 2002, ApJ, 564, 762

Frick, P., Stepanov, R., Shukurov, A., \& Sokoloff, D. 2001, MNRAS, 325, 649

Frisch, P. C. 2007, ArXiv e-prints, 0707.2970

Frisch, P. C. et al. 1999, ApJ, 525, 492

Gaensler, B. M., Haverkorn, M., Staveley-Smith, L., Dickey, J. M., McClure-Griffiths, N. M., Dickel, J. R., \& Wolleben, M. 2005, Science, 307, 1610

Gaensler, B. M., Madsen, G. J., Chatterjee, S., \& Mao, S. A. 2008, ArXiv e-prints, 0808.2550

Gieseler, U. D. J., \& Jones, T. W. 2000, A\&A, 357, 1133

Gold, B. et al. 2008, ArXiv e-prints, 0803.0715

Goldreich, P., \& Kylafis, N. D. 1981, ApJ, 243, L75

Goodman, A. A., \& Whittet, D. C. B. 1995, ApJ, 455, L181

Grogan, K., Dermott, S. F., \& Gustafson, B. A. S. 1996, ApJ, 472, 812

Grün, E., Gustafson, B., Mann, I., Baguhl, M., Morfill, G. E., Staubach, P., Taylor, A., \& Zook, H. A. 1994, A\&A, 286, 915 
Grun, E., \& Srama, R. 2006, in European Planetary Science Congress 2006, 292

Guth, A. H. 1981, Phys. Rev. D, 23, 347

Hall, J. S. 1949, Science, 109, 166

Han, J. L., Manchester, R. N., Berkhuijsen, E. M., \& Beck, R. 1997, A\&A, 322, 98

Han, J. L., Manchester, R. N., Lyne, A. G., Qiao, G. J., \& van Straten, W. 2006, ApJ, 642, 868

Han, J. L., \& Qiao, G. J. 1994, A\&A, 288, 759

Han, J. L., \& Zhang, J. S. 2007, A\&A, 464, 609

Hanbury Brown, R., Davies, R. D., \& Hazard, C. 1960, The Observatory, 80, 191

Haslam, C. G. T., Klein, U., Salter, C. J., Stoffel, H., Wilson, W. E., Cleary, M. N., Cooke, D. J., \& Thomasson, P. 1981, A\&A, 100, 209

Haslam, C. G. T., Salter, C. J., Stoffel, H., \& Wilson, W. E. 1982, A\&AS, 47, 1

Haverkorn, M. 2005, in American Institute of Physics Conference Series, Vol. 784, Magnetic Fields in the Universe: From Laboratory and Stars to Primordial Structures., ed. E. M. de Gouveia dal Pino, G. Lugones, \& A. Lazarian, 308-317

Haverkorn, M., Brown, J. C., Gaensler, B. M., \& McClure-Griffiths, N. M. 2008, ApJ, 680, 362

Haverkorn, M., Gaensler, B. M., McClure-Griffiths, N. M., Dickey, J. M., \& Green, A. J. 2006 , ApJS, 167, 230

Heiles, C. 1995, in Astronomical Society of the Pacific Conference Series, Vol. 80, The Physics of the Interstellar Medium and Intergalactic Medium, ed. A. Ferrara, C. F. McKee, C. Heiles, \& P. R. Shapiro, 507

Heiles, C. 1996, in Astronomical Society of the Pacific Conference Series, Vol. 97, Polarimetry of the Interstellar Medium, ed. W. G. Roberge \& D. C. B. Whittet, 457

Heiles, C. 2000, AJ, 119, 923

Heitsch, F., Zweibel, E. G., Mac Low, M.-M., Li, P., \& Norman, M. L. 2001, ApJ, 561, 800

Hiltner, W. A. 1949, ApJ, 109, 471

Hinshaw, G. et al. 2007, ApJS, 170, 288

- 2008, ArXiv e-prints, 0803.0732

Hirata, C. M., \& Seljak, U. 2003, Phys. Rev. D, 68, 083002

Hoang, T., \& Lazarian, A. 2008, ArXiv e-prints, 0801.0266

Hu, W., \& Okamoto, T. 2002, ApJ, 574, 566

Jansson, R., Farrar, G. R., Waelkens, A., \& Ensslin, T. A. 2007, ArXiv e-prints, 0708.2714

Jenkins, E. B. 2004, in Origin and Evolution of the Elements, ed. McWilliam \& Rauch, 336

Johnston, S. et al. 2007, Publications of the Astronomical Society of Australia, 24, 174

Jones, R. V., \& Spitzer, L. J. 1967, ApJ, 147, 943

Kim, E.-J., Olinto, A. V., \& Rosner, R. 1996, ApJ, 468, 28

Kim, S.-H., \& Martin, P. G. 1995, ApJ, 444, 293 
Kimura, H., \& Mann, I. 1998, ApJ, 499, 454

Kogut, A. et al. 2007, ApJ, 665, 355

Komatsu, E. et al. 2008, ArXiv e-prints, 0803.0547

Krüger, H., \& Grün, E. 2008, ArXiv e-prints, 0802.3787

La Porta, L., Burigana, C., Reich, W., \& Reich, P. 2008, A\&A, 479, 641

Landgraf, M. 2000, J. Geophys. Res., 105, 10303

Landgraf, M., Augustsson, K., Grün, E., \& Gustafson, B. A. S. 1999, Science, 286, 2319

Landgraf, M., Baggaley, W. J., Grün, E., Krüger, H., \& Linkert, G. 2000, J. Geophys. Res., 105, 10343

Landgraf, M., Krüger, H., Altobelli, N., \& Grün, E. 2003, Journal of Geophysical Research (Space Physics), 108, 8030

Lazarian, A. 1994, MNRAS, 268, 713

-. 1995, MNRAS, 277, 1235

- 1997, MNRAS, 288, 609

- 2007, Journal of Quantitative Spectroscopy and Radiative Transfer, 106, 225

Lazarian, A., \& Draine, B. T. 1997, ApJ, 487, 248

- 2000, ApJ, 536, L15

Lazarian, A., \& Hoang, T. 2007, MNRAS, 378, 910

Lazarian, A., \& Roberge, W. G. 1997, ApJ, 484, 230

Li, Z., Wheeler, J. C., Bash, F. N., \& Jefferys, W. H. 1991, ApJ, 378, 93

Magalhães, A. M. et al. 2005, in Astronomical Society of the Pacific Conference Series, Vol. 343, Astronomical Polarimetry: Current Status and Future Directions, ed. A. Adamson, C. Aspin, C. Davis, \& T. Fujiyoshi, 305

Martin, P. G. 1995, ApJ, 445, L63

Martin, P. G. et al. 1992, ApJ, 392, 691

Martin, P. G., Clayton, G. C., \& Wolff, M. J. 1999, ApJ, 510, 905

Mason, B., Robishaw, T., \& Finkbeiner, D. 2008, in Astronomical Society of the Pacific Conference Series, Vol. 395, Frontiers of Astrophysics: A Celebration of NRAO's 50th Anniversary, ed. A. H. Bridle, J. J. Condon, \& G. C. Hunt, 373

Mathis, J. S. 1986, ApJ, 308, 281

Mathis, J. S., Mezger, P. G., \& Panagia, N. 1983, A\&A, 128, 212

McClure-Griffiths, N. M., Dickey, J. M., Gaensler, B. M., Green, A. J., Haverkorn, M., \& Strasser, S. 2005, ApJS, 158,178

Men, H., Ferrière, K., \& Han, J. L. 2008, A\&A, 486, 819

Minter, A. H., \& Spangler, S. R. 1996, ApJ, 458, 194

Miville-Deschenes, M.-A., Ysard, N., Lavabre, A., Ponthieu, N., Macias-Perez, J. F., Aumont, J., \& Bernard, J. P. 2008, ArXiv e-prints, 0802.3345 
Mukai, T. 1981, A\&A, 99, 1

Nishiyama, S. et al. 2008, ArXiv e-prints, 0809.3089

Noutsos, A., Johnston, S., Kramer, M., \& Karastergiou, A. 2008, MNRAS, 386, 1881

Ohno, H., \& Shibata, S. 1993, MNRAS, 262, 953

Page, L. et al. 2007, ApJS, 170, 335

Pearson, T. J. 2007, in Bulletin of the American Astronomical Society, Vol. 38, 883

Peterson, J. D., \& Webber, W. R. 2002, ApJ, 575, 217

Phillipps, S., Kearsey, S., Osborne, J. L., Haslam, C. G. T., \& Stoffel, H. 1981, A\&A, 98, 286

Ponthieu, N. et al. 2005, A\&A, 444, 327

Purcell, E. M. 1979, ApJ, 231, 404

Quireza, C., Rood, R. T., Bania, T. M., Balser, D. S., \& Maciel, W. J. 2006, ApJ, 653, 1226

Rand, R. J., \& Kulkarni, S. R. 1989, ApJ, 343, 760

Rand, R. J., \& Lyne, A. G. 1994, MNRAS, 268, 497

Ratcliffe, J. A. 1959, The Magneto-Ionic Theory and Its Applications to the Ionosphere (Cambridge University Press)

Reynolds, R. J., Haffner, L. M., \& Tufte, S. L. 1999, ApJ, 525, L21

Roberge, W. G., Degraff, T. A., \& Flaherty, J. E. 1993, ApJ, 418, 287

Roberge, W. G., \& Lazarian, A. 1999, MNRAS, 305, 615

Rybicki, G. B., \& Lightman, A. P. 1979, Radiative Processes in Astrophysics (New York, WileyInterscience)

Schlegel, D. J., Finkbeiner, D. P., \& Davis, M. 1998, ApJ, 500, 525

Serkowski, K. 1973, in Interstellar Dust and Related Topics, ed. J. M. Greenberg \& H. C. van de Hulst, IAU Symposium 52, 145

Simard-Normandin, M., \& Kronberg, P. P. 1979, Nature, 279, 115

-. 1980, ApJ, 242, 74

Slavin, J. D., \& Frisch, P. C. 2008, A\&A, 491, 53

Smith, K. M. et al. 2008, ArXiv e-prints, 0811.3916

Smoot, G. F. et al. 1992, ApJ, 396, L1

Spitzer, Jr., L., \& McGlynn, T. A. 1979, ApJ, 231, 417

Srama, R. et al. 2008, Experimental Astronomy, 35

Strong, A. W., Moskalenko, I. V., \& Ptuskin, V. S. 2007, Annual Review of Nuclear and Particle Science, 57,285

Strong, A. W., Moskalenko, I. V., \& Reimer, O. 2000, ApJ, 537, 763

Sun, X. H., Reich, W., Waelkens, A., \& Enßlin, T. A. 2008, A\&A, 477, 573

Taylor, A. R. et al. 2003, AJ, 125, 3145

Tegmark, M., \& Efstathiou, G. 1996, MNRAS, 281, 1297 
Thomson, R. C., \& Nelson, A. H. 1980, MNRAS, 191, 863

Vaillancourt, J. E. 2007, in EAS Publications Series, Vol. 23, Sky Polarisation at Far-Infrared to Radio Wavelengths, ed. M.-A. Miville-Deschênes \& F. Boulanger, 147-164

Vallée, J. P. 2002, ApJ, 566, 261

Waelkens, A., Jaffe, T., Reinecke, M., Kitaura, F. S., \& Ensslin, T. A. 2008, ArXiv e-prints, 0807.2262

Weingartner, J. C., \& Draine, B. T. 2003, ApJ, 589, 289

Weisberg, J. M., Cordes, J. M., Kuan, B., Devine, K. E., Green, J. T., \& Backer, D. C. 2004, ApJS, 150,317

Whittet, D. C. B. 2003, Dust in the Galactic Environment (Bristol: IOP Publishing)

Whittet, D. C. B., Hough, J. H., Lazarian, A., \& Hoang, T. 2008, ApJ, 674, 304

Witte, M. 2004, A\&A, 426, 835

Wolleben, M. 2007, ApJ, 664, 349

Wolleben, M., Landecker, T. L., Reich, W., \& Wielebinski, R. 2006, A\&A, 448, 411

Yusef-Zadeh, F., Roberts, D. A., Goss, W. M., Frail, D. A., \& Green, A. J. 1996, ApJ, 466, L25

Zaldarriaga, M. et al. 2008, ArXiv e-prints, 0811.3918

Zweibel, E. G., \& Heiles, C. 1997, Nature, 385, 131 